\title{
Multi-leg one-loop massive amplitudes from integrand reduction via Laurent expansion
}

\author{
Hans van Deurzen, ${ }^{a}$ Gionata Luisoni, ${ }^{a}$ Pierpaolo Mastrolia, ${ }^{a, b}$ Edoardo Mirabella, ${ }^{a}$ \\ Giovanni Ossola ${ }^{c, d}$ and Tiziano Peraro ${ }^{a}$ \\ ${ }^{a}$ Max-Planck Insitut für Physik, \\ Föhringer Ring, 6, D-80805 München, Germany \\ ${ }^{b}$ Dipartimento di Fisica e Astronomia, Università di Padova, and INFN Sezione di Padova, \\ via Marzolo 8, 35131 Padova, Italy \\ ${ }^{c}$ Physics Department, New York City College of Technology, The City University of New York, \\ 300 Jay Street Brooklyn, NY 11201, U.S.A. \\ ${ }^{d}$ The Graduate School and University Center, The City University of New York, \\ 365 Fifth Avenue, New York, NY 10016, U.S.A \\ E-mail: hdeurzen@mpp.mpg.de, luisonig@mpp.mpg.de, \\ pierpaolo.mastrolia@cern.ch, mirabell@mpp.mpg.de, \\ GOssola@citytech. cuny.edu, peraro@mpp.mpg.de
}

ABSTRACT: We present the application of a novel reduction technique for one-loop scattering amplitudes based on the combination of the integrand reduction and Laurent expansion. We describe the general features of its implementation in the computer code NINJA, and its interface to GoSAm. We apply the new reduction to a series of selected processes involving massive particles, from six to eight legs.

KEYWORDS: NLO Computations

ARXIV EPRINT: 1312.6678 


\section{Contents}

1 Introduction $\quad 2$

2 Reduction algorithm - integrand reduction via Laurent expansion $\quad 4$

2.1 Integrand and integral decomposition 4

$\begin{array}{lll}2.2 & \text { Scattering amplitudes via Laurent expansion } & 6\end{array}$

$\begin{array}{lll}2.3 & \text { The } \mathrm{C}++ \text { library Ninja } & 10\end{array}$

3 Interfacing NINJA with GOSAM $\quad 12$

4 Precision tests 13

5 Applications to massive amplitudes $\quad \mathbf{1 7}$

$\begin{array}{ll}5.1 \quad p p \rightarrow W+3 \text { jets } & 18\end{array}$

$\begin{array}{ll}5.2 p p \rightarrow Z+3 \text { jets } & 19\end{array}$

$\begin{array}{ll}5.3 p p \rightarrow Z Z Z+1 \text { jet } & 19\end{array}$

$5.4 p p \rightarrow W W Z+1$ jet $\quad 20$

$5.5 p p \rightarrow W Z Z+1$ jet $\quad 20$

$5.6 \quad p p \rightarrow W W W+1$ jet 21

$5.7 p p \rightarrow Z Z Z Z \quad 21$

$5.8 p p \rightarrow W W W W \quad 22$

$5.9 p p \rightarrow t \bar{t} b \bar{b} \quad 22$

$5.10 p p \rightarrow t \bar{t}+2$ jets $\quad 23$

$5.11 p p \rightarrow Z b \bar{b}+1$ jet 24

$5.12 p p \rightarrow W b \bar{b}+1$ jet $\quad 24$

$5.13 p p \rightarrow W b \bar{b}+2$ jets $\quad 25$

$5.14 p p \rightarrow W W b \bar{b} \quad 26$

$5.15 p p \rightarrow W W b \bar{b}+1$ jet $\quad 27$

$5.16 p p \rightarrow H+3$ jets 28

$5.17 p p \rightarrow Z t \bar{t}+1$ jet $\quad 28$

$5.18 p p \rightarrow H t \bar{t}+1$ jet $\quad 29$

$5.19 p p \rightarrow H+3$ jets $(\mathrm{VBF}) \quad 30$

$5.20 p p \rightarrow H+4$ jets $(\mathrm{VBF}) \quad 30$

$5.21 p p \rightarrow H+5$ jets $(\mathrm{VBF})$

6 Conclusions $\quad 32$ 


\section{Introduction}

Scattering amplitudes in quantum field theories are analytic functions of the kinematic variables of the interacting particles, hence they can be determined by studying the structure of their singularities.

The multi-particle factorization properties of the amplitudes become transparent when internal particles go on their mass-shell [1,2]. These configurations correspond to poles of the amplitude and the investigation of the general structure of the residues corresponding to multi-particle factorization channel turns out to be of particular interest. Indeed the knowledge of such structure has been fundamental for discovering new relations fulfilled by scattering amplitudes, such as the BCFW recurrence relation [3] and its link to the leading singularity of one-loop amplitudes [4]. The systematic classification of the residues, for all the poles corresponding to the quadruple, triple, double, and single cuts, has been achieved, in four dimensions, by employing integrand-reduction methods $[5,6]$. The latter led to the OPP integrand-decomposition formula for one-loop integrals [6], which allows one to write each residue as a linear combination of process-independent polynomials multiplied by process-dependent coefficients.

These results provided a deeper understanding of the structure of scattering amplitudes and have shown the underlying simplicity beneath the rich mathematical structure of quantum field theory. Moreover, they provided the theoretical framework for the development of efficient computational algorithms for one-loop calculations in perturbation theory, which have been implemented in various automated codes [7-16] improving the state-of-the art of the predictions at the next-to-leading order accuracy [17-50].

Recently, the classification of the structure of the residues has been obtained in a more general and elegant form within the framework of multivariate polynomial division and algebraic geometry [51,52]. The use of these techniques proved that the integrand decomposition, originally formulated for one-loop amplitudes, is applicable at any order in perturbation theory, irrespective of the complexity of the topology of the diagrams involved. An iterative integrand-recursion formula, based on successive divisions of the numerators modulo the Gröbner basis of the ideals generated by the cut denominators, can provide the form of the residues at all multi-particle poles for arbitrary amplitudes, independently of the number of loops. Extensions of the integrand reduction method beyond one-loop, initiated in $[53,54]$ and then systematized within the language of algebraic geometry [51, 52] have recently become the topic of several studies [55-60], thus providing a new direction in the study of multi-loop amplitudes.

In the context of the integrand reduction, the process-dependent coefficients can be numerically determined by solving a system of algebraic equations that are obtained by evaluating the numerator of the integrand at explicit values of the loop momentum [6]. The system becomes triangular if one evaluates the numerator at the multiple cuts, i.e. at the set of complex values of the integration momentum for which a given set of propagators vanish. The extraction of all coefficients via polynomial fitting has been implemented in publicly available codes performing integrand decomposition, such as CuTTools [61] and SAmURAI [62]. These algorithms do not requires any specific recipe for the generation of 
the numerator function, which can be performed by using traditional Feynman diagrams, by means of recursive relations, or by gluing tree-level sub-amplitudes, as in unitaritybased methods.

The code CutTools implements the four-dimensional integrand-reduction algorithm [63-65], in which the cut-constructible term and the rational term are necessarily computed separately. The latter escapes four-dimensional integrand reduction and has to be computed using other methods, e.g. by using ad hoc tree-level Feynman rules [65-72].

Significant improvements were achieved with the $d$-dimensional extension of integrandreduction methods [73-75], which expose a richer polynomial structure of the integrand and allows for the combined determination of both cut-constructible and rational terms at once. This idea of performing unitarity-cuts in $d$-dimension was the basis for the development of SAMURAI, which extends the OPP polynomial structures to include an explicit dependence on the $(d-4)$-dimensional parameter needed for the automated computation of the full rational term. Moreover, it includes the parametrization of the residue of the quintuplecut [76] and implements the numerical sampling via Discrete Fourier Transform [64].

The integrand decomposition was originally developed for renormalizable gauge theories, where, at one-loop, the rank of the numerator cannot be larger than the number of external legs. The reduction of diagrams where the rank can be higher, as required for example when computing $p p \rightarrow H+2,3$ jets in gluon fusion in the large top-mass limit $[42,44]$, demands an extension of the algorithm to accommodate the richer monomial structures of the residues. This extension has been implemented in SAmURAI, together with the corresponding sampling required to fit all the coefficients [77-79].

More recently, elaborating on the the techniques proposed in [80, 81], a different approach to the $d$-dimensional integrand-reduction method was proposed [78]. The key point of this method is to extract the coefficients more efficiently by performing a Laurent expansion of the integrand. The method is general and relies only on the knowledge of the explicit dependence of the numerator on the loop momentum.

In general, when the multiple-cut conditions do not fully constrain the loop momentum, the solutions are still functions of some free parameters, possibly the components of the momentum which are not frozen by the cut conditions. The integrand-reduction algorithms implemented in the codes $[61,62]$ require to solve a system of equations obtained by sampling on those free parameters. The system is triangular thus the coefficients of a given residue can be computed only after subtracting all the non-vanishing contributions coming from higher-point residues.

The key observation suggested in ref. [78] is that the reduction algorithm can be simplified by exploiting the universal structure of the residues, hence of their asymptotic expansion. Indeed, by performing a Laurent expansion with respect to one of the free parameters which appear in the solutions of the cut, both the integrand and the subtraction terms exhibit the same polynomial behavior of the residue. Moreover, the contributions coming from the subtracted terms can be implemented as corrections at the coefficient level, hence replacing the subtractions at the integrand level of the original algorithm. The parametric form of these corrections can be computed once and for all, in terms of a subset of the higher-point coefficients. This method significantly reduces the number 
of coefficients entering each subtracted term, in particular boxes and pentagons decouple from the computation of lower-points coefficients.

If either the analytic expression of the integrand or the tensor structure of the numerator is known, this procedure can be implemented in a semi-numerical algorithm. Indeed, the coefficients of the Laurent expansion of a rational function can be computed, either analytically or numerically, by performing a polynomial division between the numerator and the (uncut) denominators.

The scope of the present paper is to review the main features of the novel reduction algorithm and demonstrate its performance on a selection of challenging calculations of scattering amplitudes with massive bosons and quarks, involving six, seven, and eight particles. The integrand-reduction via Laurent expansion has been implemented in the C++ library NinJa [82], and interfaced to the GoSAM framework [12] for the evaluation of virtual one-loop scattering amplitudes. The cleaner and lighter system-solving strategy, which deals with a diagonal system instead of a triangular one, and which substitutes the polynomial subtractions with coefficients corrections, turns into net gains in terms of both numerical accuracy and computing speed. We recall that the new library has been recently used in the evaluation of NLO QCD corrections to $p p \rightarrow t \bar{t} H j$ [45].

The paper is organized as follows. In section 2, we discuss the theoretical foundations of the integrand decomposition via Laurent expansion, and its implementation in an algorithm for the reduction of one-loop amplitudes. The description of the interface between NiNJA and GoSAm for automated one-loop calculation is discussed in section 3. Section 4 is devoted to a detailed study of the precision and of the computational performance of the novel framework, which shows a significant improvement with respect to the standard algorithms. Applications of the GoSAM+NINJA framework to the evaluation of NLO QCD virtual correction to several multi-leg massive processes are shown in section 5 .

\section{Reduction algorithm - integrand reduction via Laurent expansion}

In this section we describe the Laurent-expansion method for the integrand reduction of one-loop amplitudes as implemented in the $\mathrm{C}++$ library NinJA.

\subsection{Integrand and integral decomposition}

An $n$-point one-loop amplitude can be written as a linear combination of contributions $\mathcal{M}_{1 \cdots n}$ of the form

$$
\mathcal{M}_{1 \cdots n}=\int d^{d} \bar{q} \mathcal{I}_{1 \cdots n}=\int d^{d} \bar{q} \frac{\mathcal{N}(\bar{q})}{D_{1} \cdots D_{n}}
$$

where $\mathcal{N}(\bar{q})$ is a process-dependent polynomial numerator in the components of the $d=$ $(4-2 \epsilon)$-dimensional loop momentum $\bar{q}$. The latter can be decomposed as follows,

$$
\bar{q}=\not q+\mu, \quad \bar{q}^{2}=q^{2}-\mu^{2},
$$

in terms of its 4 -dimensional component, $q$, and $\mu^{2}$ which encodes its $(-2 \epsilon)$-dimensional components. The denominators $D_{i}$ are quadratic polynomials in $\bar{q}$ and correspond to 
Feynman loop propagators,

$$
D_{i}=\left(\bar{q}+p_{i}\right)^{2}-m_{i}^{2}
$$

Every one-loop integrand in $d$ dimensions can be decomposed as $[6,73]$

$$
\mathcal{I}_{1 \ldots n} \equiv \frac{\mathcal{N}_{1 \cdots n}}{D_{1} \ldots D_{n}}=\sum_{k=1}^{5} \sum_{\left\{i_{1}, \cdots, i_{k}\right\}} \frac{\Delta_{i_{1} \cdots i_{k}}}{D_{i_{1}} \cdots D_{i_{k}}},
$$

where the $\Delta_{i_{1} \cdots i_{k}}$ are irreducible polynomial residues, i.e. polynomials which do not contain any term proportional to the corresponding loop denominators $D_{i_{1}}, \ldots, D_{i_{k}}$. The second sum in eq. (2.4) runs over all unordered selections without repetition of the $k$ indices $\left\{i_{1}, \cdots, i_{k}\right\}$.

For any set of denominators $D_{i_{1}}, \ldots, D_{i_{k}}$ with $k \leq 5$, one can choose a 4-dimensional basis of momenta $\mathcal{E}=\left\{e_{1}, e_{2}, e_{3}, e_{4}\right\}$ which satisfies the following normalization conditions

$$
e_{1} \cdot e_{2}=1, \quad e_{3}^{2}=e_{4}^{2}=\delta_{k 4}, \quad e_{3} \cdot e_{4}=-\left(1-\delta_{k 4}\right),
$$

while all the other scalar products vanish. In addition, for $k=4$ we choose the basis such that $e_{4}$ is orthogonal to the external legs of the sub-diagram identified by the set of denominators in consideration. Similarly, for $k=2,3$ we choose both $e_{3}$ and $e_{4}$ to be orthogonal to the external legs of the corresponding sub-diagram. With this choices of momentum basis, the numerator and the denominators can be written as polynomials in the coordinates $\mathbf{z} \equiv\left(z_{1}, z_{2}, z_{3}, z_{4}, z_{5}\right)=\left(x_{1}, x_{2}, x_{3}, x_{4}, \mu^{2}\right)$. The variables $x_{i}$ are the components of $q$ with respect to the basis $\mathcal{E}$,

$$
q^{\nu}=-p_{i_{1}}^{\nu}+x_{1} e_{1}^{\nu}+x_{2} e_{2}^{\nu}+x_{3} e_{3}^{\nu}+x_{4} e_{4}^{\nu}
$$

More explicitly, the numerator is a polynomial in the components of $q$ and $\mu^{2}$

$$
\mathcal{N}(\bar{q})=\mathcal{N}\left(q, \mu^{2}\right)=\mathcal{N}\left(x_{1}, x_{2}, x_{3}, x_{4}, \mu^{2}\right)=\mathcal{N}(\mathbf{z})
$$

The coordinates $x_{i}$ can also be written as scalar products,

$$
\begin{aligned}
& x_{1}=\left(q+p_{i_{1}}\right) \cdot e_{2} \\
& x_{2}=\left(q+p_{i_{1}}\right) \cdot e_{1} \\
& x_{3}=-\left(\left(q+p_{i_{1}}\right) \cdot e_{4}\right)\left(1-\delta_{k 4}\right)+\left(\left(q+p_{i_{1}}\right) \cdot e_{3}\right) \delta_{k 4} \\
& x_{4}=-\left(\left(q+p_{i_{1}}\right) \cdot e_{3}\right)\left(1-\delta_{k 4}\right)+\left(\left(q+p_{i_{1}}\right) \cdot e_{4}\right) \delta_{k 4} .
\end{aligned}
$$

With these definitions, one can show $[6,51,73]$ that the most general parametric form 
of a residue in a renormalizable theory is

$$
\begin{aligned}
\Delta_{i_{1} i_{2} i_{3} i_{4} i_{5}=}= & c_{0}^{\left(i_{1} i_{2} i_{3} i_{4} i_{5}\right)} \mu^{2} \\
\Delta_{i_{1} i_{2} i_{3} i_{4}}= & c_{0}^{\left(i_{1} i_{2} i_{3} i_{4}\right)}+c_{1}^{\left(i_{1} i_{2} i_{3} i_{4}\right)} x_{4}+\mu^{2}\left(c_{2}^{\left(i_{1} i_{2} i_{3} i_{4}\right)}+c_{3}^{\left(i_{1} i_{2} i_{3} i_{4}\right)} x_{4}+\mu^{2} c_{4}^{\left(i_{1} i_{2} i_{3} i_{4}\right)}\right) \\
\Delta_{i_{1} i_{2} i_{3}}= & c_{0}^{\left(i_{1} i_{2} i_{3}\right)}+c_{1}^{\left(i_{1} i_{2} i_{3}\right)} x_{3}+c_{2}^{\left(i_{1} i_{2} i_{3}\right)} x_{3}^{2}+c_{3}^{\left(i_{1} i_{2} i_{3}\right)} x_{3}^{3}+c_{4}^{\left(i_{1} i_{2} i_{3}\right)} x_{4}+c_{5}^{\left(i_{1} i_{2} i_{3}\right)} x_{4}^{2} \\
& +c_{6}^{\left(i_{1} i_{2} i_{3}\right)} x_{4}^{3}+\mu^{2}\left(c_{7}^{\left(i_{1} i_{2} i_{3}\right)}+c_{8}^{\left(i_{1} i_{2} i_{3}\right)} x_{3}+c_{9}^{\left(i_{1} i_{2} i_{3}\right)} x_{4}\right) \\
\Delta_{i_{1} i_{2}}= & c_{0}^{\left(i_{1} i_{2}\right)}+c_{1}^{\left(i_{1} i_{2}\right)} x_{2}+c_{2}^{\left(i_{1} i_{2}\right)} x_{3}+c_{3}^{\left(i_{1} i_{2}\right)} x_{4}+c_{4}^{\left(i_{1} i_{2}\right)} x_{2}^{2}+c_{5}^{\left(i_{1} i_{2}\right)} x_{3}^{2} \\
& +c_{6}^{\left(i_{1} i_{2}\right)} x_{4}^{2}+c_{7}^{\left(i_{1} i_{2}\right)} x_{2} x_{3}+c_{8}^{\left(i_{1} i_{2}\right)} x_{2} x_{4}+c_{9}^{\left(i_{1} i_{2}\right)} \mu^{2} \\
\Delta_{i_{1}}= & c_{0}^{\left(i_{1}\right)}+c_{1}^{\left(i_{1}\right)} x_{1}+c_{2}^{\left(i_{1}\right)} x_{2}+c_{3}^{\left(i_{1}\right)} x_{3}+c_{4}^{\left(i_{1}\right)} x_{4} .
\end{aligned}
$$

This parametric form can also be extended to non-renormalizable and effective theories, where the rank of the numerator can be larger than the number of loop propagators [78]. Most of the term appearing in eq. (2.9) vanish after integration, i.e. they are spurious. The non-spurious coefficients, instead, appear in the final result which expresses the amplitude $\mathcal{M}_{1 \cdots n}$ as a linear combination of known Master Integrals,

$$
\begin{aligned}
\mathcal{M}_{1 \cdots n}= & \sum_{\left\{i_{1}, i_{2}, i_{3}, i_{4}\right\}}\left\{c_{0}^{\left(i_{1} i_{2} i_{3} i_{4}\right)} I_{i_{1} i_{2} i_{3} i_{4}}+c_{4}^{\left(i_{1} i_{2} i_{3} i_{4}\right)} I_{i_{1} i_{2} i_{3} i_{4}}\left[\mu^{4}\right]\right\} \\
& +\sum_{\left\{i_{1}, i_{2}, i_{3}\right\}}^{n-1}\left\{c_{0}^{\left(i_{1} i_{2} i_{3}\right)} I_{i_{1} i_{2} i_{3}}+c_{7}^{\left(i_{1} i_{2} i_{3}\right)} I_{\left.i_{1} i_{2} i_{3}\left[\mu^{2}\right]\right\}}\right\} \\
& +\sum_{\left\{i_{1}, i_{2}\right\}}\left\{c_{0}^{\left(i_{1} i_{2}\right)} I_{i_{1} i_{2}}+c_{1}^{\left(i_{1} i_{2}\right)} I_{i_{1} i_{2}}\left[\left(q+p_{i_{1}}\right) \cdot e_{2}\right]\right. \\
& \left.\quad+c_{2}^{\left(i_{1} i_{2}\right)} I_{i_{1} i_{2}}\left[\left(\left(q+p_{i_{1}}\right) \cdot e_{2}\right)^{2}\right]+c_{9}^{\left(i_{1} i_{2}\right)} I_{i_{1} i_{2}}\left[\mu^{2}\right]\right\} \\
& +\sum_{i_{1}} c_{0}^{\left(i_{1}\right)} I_{i_{1}},
\end{aligned}
$$

where

$$
I_{i_{1} \cdots i_{k}}[\alpha] \equiv \int d^{d} \bar{q} \frac{\alpha}{D_{i_{1}} \cdots D_{i_{k}}}, \quad I_{i_{1} \cdots i_{k}} \equiv I_{i_{1} \cdots i_{k}}[1] .
$$

The problem of the computation of any one-loop amplitude can therefore be reduced to the problem of the determination of the coefficients of the Master Integrals appearing in eq. (2.10), which in turn can be identified with a subset of the coefficients of the parametric residues in eq. (2.9).

\subsection{Scattering amplitudes via Laurent expansion}

In ref. [78], elaborating on the the techniques proposed in [80, 81], an improved version of the integrand-reduction method for one-loop amplitudes was presented. This method allows, whenever the analytic dependence of the integrand on the loop momentum is known, 
to extract the unknown coefficients of the residues $\Delta_{i_{1} \cdots i_{k}}$ by performing a Laurent expansion of the integrand with respect to one of the free loop components which are not constrained by the corresponding on-shell conditions $D_{i_{1}}=\cdots=D_{i_{k}}=0$.

Within the original integrand reduction algorithm [61,62, 64], the determination of these unknown coefficients requires: i) to sample the numerator on a finite subset of the onshell solutions; ii) to subtract from the integrand the non-vanishing contributions coming from higher-point residues; and iii) to solve the resulting linear system of equations.

With the Laurent-expansion approach, since in the asymptotic limit both the integrand and the higher-point subtractions exhibit the same polynomial behavior as the residue, one can instead identify the unknown coefficients with the ones of the expansion of the integrand, corrected by the contributions coming from higher-point residues. In other words, the system of equations for the coefficients becomes diagonal and the subtractions of higher-point contributions can be implemented as corrections at the coefficient level which replace the subtractions at the integrand level of the original algorithm. Because of the universal structure of the residues, the parametric form of these corrections can be computed once and for all, in terms of a subset of the higher-point coefficients. This also allows to significantly reduce the number of coefficients entering in each subtraction. For instance, box and pentagons do not affect at all the computation of lower-points residues. In the followings, we describe in more detail how to determine the needed coefficients of each residue.

Pentagons. Pentagons contributions are spurious, i.e. they do not appear in the integrated result. In the original integrand reduction algorithm their computation is nevertheless needed, in order to implement the corresponding subtractions at the integrand level. Within the Laurent expansion approach, since the subtraction terms of five-point residues always vanish in the asymptotic limit, their computation is instead not needed and can be skipped.

Boxes. The coefficient $c_{0}$ of the box contributions can be determined via 4-dimensional quadruple cuts [4]. In four dimensions (i.e. $\bar{q}=q, \mu^{2}=0$ ) a quadruple cut $D_{i_{1}}=\cdots=$ $D_{i_{4}}=0$ has two solutions, $q_{+}$and $q_{-}$. The coefficient $c_{0}$ can be expressed in terms of these solutions as

$$
c_{0}^{\left(i_{1} i_{2} i_{3} i_{4}\right)}=\frac{1}{2}\left(\left.\frac{\mathcal{N}(q)}{\prod_{j \neq i_{1}, i_{2}, i_{3}, i_{4}} D_{j}}\right|_{q=q_{+}}+\left.\frac{\mathcal{N}(q)}{\prod_{j \neq i_{1}, i_{2}, i_{3}, i_{4}} D_{j}}\right|_{q=q_{-}}\right) .
$$

The coefficient $c_{4}$ can be found by evaluating the integrand on $d$-dimensional quadruple cuts in the asymptotic limit $\mu^{2} \rightarrow \infty$ [81]. A $d$-dimensional quadruple cut has an infinite number of solutions which can be parametrized by the extra-dimensional variable $\mu^{2}$. These solutions become particularly simple in the limit of large $\mu^{2}$, namely

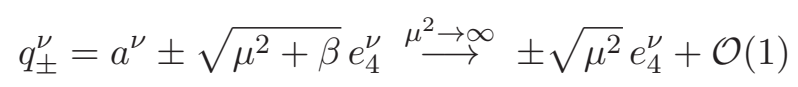

where the vector $a^{\nu}$ and the constant $\beta$ are fixed by the cut conditions. The coefficient $c_{4}$, when non-vanishing, can be found in this limit as the leading term of the expansion of the 
integrand

$$
\frac{\mathcal{N}\left(q, \mu^{2}\right)}{\prod_{j \neq i_{1}, i_{2}, i_{3}, i_{4}} D_{j}} \stackrel{\mu^{2} \rightarrow \infty}{\longrightarrow} c_{4}^{\left(i_{1} i_{2} i_{3} i_{4}\right)} \mu^{4}+\mathcal{O}\left(\mu^{3}\right) .
$$

The other coefficients of the boxes are spurious and their computation can be avoided.

Triangles. The residues of the triangle contributions can be determined by evaluating the integrand on the solutions of $d$-dimensional triple cuts [80], which can be parametrized by the extra-dimensional variable $\mu^{2}$ and another parameter $t$,

$$
q_{+}=a^{\nu}+t e_{3}^{\nu}+\frac{\beta+\mu^{2}}{2 t} e_{4}^{\nu}, \quad q_{-}=a^{\nu}+\frac{\beta+\mu^{2}}{2 t} e_{3}^{\nu}+t e_{4}^{\nu},
$$

where the vector $a^{\nu}$ and the constant $\beta$ are fixed by the cut conditions $D_{i_{1}}=D_{i_{2}}=$ $D_{i_{3}}=0$. On these solutions, the integrand generally receives contributions from the residue of the triple cut $\Delta_{i_{1} i_{2} i_{3}}$, as well as from the boxes and pentagons which share the three cut denominators. However, after taking the asymptotic expansion at large $t$ and dropping the terms which vanish in this limit, the pentagon contributions vanish, while the box contributions are constant in $t$ but vanish when taking the average between the parametrizations $q_{+}$and $q_{-}$of eq. (2.14). More explicitly,

$$
\begin{aligned}
\frac{\mathcal{N}\left(q_{ \pm}, \mu^{2}\right)}{\prod_{j \neq i_{1}, i_{2}, i_{3}} D_{j}} & =\Delta_{i_{1} i_{2} i_{3}}+\sum_{j} \frac{\Delta_{i_{1} i_{2} i_{3} j}}{D_{j}}+\sum_{j k} \frac{\Delta_{i_{1} i_{2} i_{3} j k}}{D_{j} D_{k}} \\
& =\Delta_{i_{1} i_{2} i_{3}}+d_{1}^{ \pm}+d_{2}^{ \pm} \mu^{2}+\mathcal{O}(1 / t), \quad \text { with } d_{i}^{+}+d_{i}^{-}=0 .
\end{aligned}
$$

Moreover, even though the integrand is a rational function, in this asymptotic limit it exhibits the same polynomial behavior as the expansion of the residue $\Delta_{i_{1} i_{2} i_{3}}$,

$$
\begin{aligned}
\frac{\mathcal{N}\left(q_{+}, \mu^{2}\right)}{\prod_{j \neq i_{1}, i_{2}, i_{3}} D_{j}}= & n_{0}^{+}+n_{7}^{+} \mu^{2}-\left(n_{4}+n_{9} \mu^{2}\right) t+n_{5} t^{2}+n_{6} t^{3}+\mathcal{O}(1 / t) \\
\frac{\mathcal{N}\left(q_{-}, \mu^{2}\right)}{\prod_{j \neq i_{1}, i_{2}, i_{3}} D_{j}}= & n_{0}^{-}+n_{7}^{-} \mu^{2}-\left(n_{1}+n_{8} \mu^{2}\right) t+n_{2} t^{2}+n_{3} t^{3}+\mathcal{O}(1 / t) \\
\Delta_{i_{1} i_{2} i_{3}}\left(q_{+}, \mu^{2}\right)= & c_{0}^{\left(i_{1} i_{2} i_{3}\right)}+c_{7}^{\left(i_{1} i_{2} i_{3}\right)} \mu^{2}-\left(c_{4}^{\left(i_{1} i_{2} i_{3}\right)}+c_{9}^{\left(i_{1} i_{2} i_{3}\right)} \mu^{2}\right) t \\
& +c_{5}^{\left(i_{1} i_{2} i_{3}\right)} t^{2}-c_{6}^{\left(i_{1} i_{2} i_{3}\right)} t^{3}+\mathcal{O}(1 / t) \\
\Delta_{i_{1} i_{2} i_{3}}\left(q_{-}, \mu^{2}\right)= & c_{0}^{\left(i_{1} i_{2} i_{3}\right)}+c_{7}^{\left(i_{1} i_{2} i_{3}\right)} \mu^{2}-\left(c_{1}^{\left(i_{1} i_{2} i_{3}\right)}+c_{8}^{\left(i_{1} i_{2} i_{3}\right)} \mu^{2}\right) t \\
& +c_{2}^{\left(i_{1} i_{2} i_{3}\right)} t^{2}-c_{3}^{\left(i_{1} i_{2} i_{3}\right)} t^{3}+\mathcal{O}(1 / t) .
\end{aligned}
$$

By comparison of eqs. (2.15), (2.16) and (2.17) one gets all the triangle coefficients as

$$
c_{i}^{\left(i_{1} i_{2} i_{3}\right)}=\frac{1}{2}\left(n_{i}^{+}+n_{i}^{-}\right) \quad \text { for } i=0,7, \quad c_{i}^{\left(i_{1} i_{2} i_{3}\right)}=n_{i} \quad \text { for } i \neq 0,7 .
$$

It is worth to observe that, within the Laurent expansion approach, the determination of the 3 -point residues does not require any subtraction of higher-point terms. 
Bubbles. The on-shell solutions of a $d$-dimensional double cut $D_{i_{1}}=D_{i_{2}}=0$ can be parametrized as

$$
\begin{aligned}
& q_{+}=a_{0}^{\nu}+x a_{1}^{\nu}+t e_{3}^{\nu}+\frac{\beta_{0}+\beta_{1} x+\beta_{2} x^{2}+\mu^{2}}{2 t} e_{4}^{\nu}, \\
& q_{-}=a_{0}^{\nu}+x a_{1}^{\nu}+\frac{\beta_{0}+\beta_{1} x+\beta_{2} x^{2}+\mu^{2}}{2 t} e_{3}^{\nu}+t e_{4}^{\nu},
\end{aligned}
$$

in terms of the three free parameters $x, t$ and $\mu^{2}$, while the vectors $a_{i}^{\nu}$ and the constants $\beta_{i}$ are fixed by the on-shell conditions. After evaluating the integrand on these solutions and taking the asymptotic limit $t \rightarrow \infty$, the only non-vanishing subtraction terms come from the triangle contributions,

$$
\begin{aligned}
\frac{\mathcal{N}\left(q_{ \pm}, \mu^{2}\right)}{\prod_{j \neq i_{1}, i_{2}} D_{j}} & =\Delta_{i_{1} i_{2}}+\sum_{j} \frac{\Delta_{i_{1} i_{2} j}}{D_{j}}+\sum_{j k} \frac{\Delta_{i_{1} i_{2} j k}}{D_{j} D_{k}}+\sum_{j k l} \frac{\Delta_{i_{1} i_{2} j k l}}{D_{j} D_{k} D_{l}} \\
& =\Delta_{i_{1} i_{2}}+\sum_{j} \frac{\Delta_{i_{1} i_{2} j}}{D_{j}}+\mathcal{O}(1 / t) .
\end{aligned}
$$

The integrand and the subtraction term are rational function, but in the asymptotic limit they both have the same polynomial behavior as the residue, namely

$$
\begin{aligned}
\frac{\mathcal{N}\left(q_{+}, \mu^{2}\right)}{\prod_{j \neq i_{1}, i_{2}} D_{j}}= & n_{0}+n_{9} \mu^{2}+n_{1} x+n_{2} x^{2}-\left(n_{5}+n_{8} x\right) t+n_{6} t^{2}+\mathcal{O}(1 / t) \\
\frac{\mathcal{N}\left(q_{-}, \mu^{2}\right)}{\prod_{j \neq i_{1}, i_{2}} D_{j}}= & n_{0}+n_{9} \mu^{2}+n_{1} x+n_{2} x^{2}-\left(n_{3}+n_{7} x\right) t+n_{4} t^{2}+\mathcal{O}(1 / t) \\
\frac{\Delta_{i_{1} i_{2} j}\left(q_{+}, \mu^{2}\right)}{D_{j}}= & c_{s, 0}^{(j)}+c_{s, 9}^{(j)} \mu^{2}+c_{s, 1}^{(j)} x+c_{s, 2}^{(j)} x^{2}-\left(c_{s, 5}^{(j)}+c_{s, 8}^{(j)} x\right) t+c_{s, 6}^{(j)} t^{2}+\mathcal{O}(1 / t) \\
\frac{\Delta_{i_{1} i_{2} j}\left(q_{-}, \mu^{2}\right)}{D_{j}}= & c_{s, 0}^{(j)}+c_{s, 9}^{(j)} \mu^{2}+c_{s, 1}^{(j)} x+c_{s, 2}^{(j)} x^{2}-\left(c_{s, 3}^{(j)}+c_{s, 7}^{(j)} x\right) t+c_{s, 4}^{(j)} t^{2}+\mathcal{O}(1 / t) \\
\Delta_{i_{1} i_{2}}\left(q_{+}, \mu^{2}\right)= & c_{0}^{\left(i_{1} i_{2}\right)}+c_{9}^{\left(i_{1} i_{2}\right)} \mu^{2}+c_{1}^{\left(i_{1} i_{2}\right)} x+c_{2}^{\left(i_{1} i_{2}\right)} x^{2}-\left(c_{5}^{\left(i_{1} i_{2}\right)}+c_{8}^{\left(i_{1} i_{2}\right)} x\right) t \\
& +c_{6}^{\left(i_{1} i_{2}\right)} t^{2}+\mathcal{O}(1 / t) \\
\Delta_{i_{1} i_{2}}\left(q_{-}, \mu^{2}\right)= & c_{0}^{\left(i_{1} i_{2}\right)}+c_{9}^{\left(i_{1} i_{2}\right)} \mu^{2}+c_{1}^{\left(i_{1} i_{2}\right)} x+c_{2}^{\left(i_{1} i_{2}\right)} x^{2}-\left(c_{3}^{\left(i_{1} i_{2}\right)}+c_{7}^{\left(i_{1} i_{2}\right)} x\right) t \\
& +c_{4}^{\left(i_{1} i_{2}\right)} t^{2}+\mathcal{O}(1 / t) .
\end{aligned}
$$

The coefficients $c_{s, i}^{(j)}$ of the Laurent expansion of the subtractions terms in eqs. (2.22) can be computed once and for all as parametric functions of the triangles coefficients. Therefore, the subtraction of the triangles can be implemented as corrections to the coefficients of the expansion of the integrand. Indeed, by inserting eqs. (2.21), (2.22) and (2.23) in eq. (2.20) one gets

$$
c_{i}^{\left(i_{1} i_{2}\right)}=n_{i}-\sum_{j} c_{s, i}^{(j)} \text { for } i=0, \ldots, 9 .
$$


Tadpoles. Once the coefficients of the triangles and the bubbles are known, one can determine the non-spurious coefficient $c_{0}$ of a tadpole residue $\Delta_{i_{1}}$ by evaluating the integrand on the single cut $D_{i_{1}}=0$. One can choose 4-dimensional solutions of the form

$$
q_{+}^{\nu}=-p_{i_{1}}^{\nu}+t e_{3}^{\nu}+\frac{\beta}{2 t} e_{4}^{\nu}
$$

parametrized by the free variable $t$, while the constant $\beta$ is fixed by the cut conditions. In the asymptotic limit $t \rightarrow \infty$, only bubbles and triangles coefficients are non-vanishing,

$$
\begin{aligned}
\frac{\mathcal{N}\left(q_{+}\right)}{\prod_{j \neq i_{1}} D_{j}} & =\Delta_{i_{1}}+\sum_{j} \frac{\Delta_{i_{1} j}}{D_{j}}+\sum_{j k} \frac{\Delta_{i_{1} j k}}{D_{j} D_{k}}+\sum_{j k l} \frac{\Delta_{i_{1} j k l}}{D_{j} D_{k} D_{l}} \\
& =\Delta_{i_{1}}+\sum_{j} \frac{\Delta_{i_{1} j}}{D_{j}}+\sum_{j k} \frac{\Delta_{i_{1} j k}}{D_{j} D_{k}}+\mathcal{O}(1 / t) .
\end{aligned}
$$

Similarly to the case of the bubbles, in this limit the integrand and the subtraction terms exhibit the same polynomial behavior as the residue, i.e.

$$
\begin{aligned}
\frac{\mathcal{N}\left(q_{+}\right)}{\prod_{j \neq i_{1}} D_{j}} & =n_{0}-n_{4} t+\mathcal{O}(1 / t) \\
\frac{\Delta_{i_{1} j}\left(q_{+}\right)}{D_{j}} & =c_{s_{2}, 0}^{(j)}-c_{s_{2}, 4}^{(j)} t+\mathcal{O}(1 / t) \\
\frac{\Delta_{i_{1} j k}\left(q_{+}\right)}{D_{j} D_{k}} & =c_{s_{3}, 0}^{(j k)}-c_{s_{3}, 4}^{(j k)} t+\mathcal{O}(1 / t) \\
\Delta_{i_{1}}\left(q_{+}\right) & =c_{0}^{\left(i_{1}\right)}-c_{4}^{\left(i_{1}\right)} t .
\end{aligned}
$$

Putting everything together, we can write the coefficient of the tadpole integral as the corresponding one in the expansion of the integrand, corrected by coefficient-level subtractions

$$
c_{0}^{\left(i_{1}\right)}=n_{0}-\sum_{j} c_{s_{2}, 0}^{(j)}-\sum_{j k} c_{s_{3}, 0}^{(j k)}
$$

Once again, we observe that the subtraction terms $c_{s_{2}, 0}^{(j)}$ and $c_{s_{3}, 0}^{(j k)}$, coming from bubbles and triangles contributions respectively, are known parametric functions of the coefficients of the corresponding higher-point residues.

\subsection{The C ++ library NinJA}

The C ++ library NinJa [82] provides a semi-numerical implementation of the Laurent expansion method described above. Since the integrand is a rational function of the loop variables, its Laurent expansion is performed via a simplified polynomial division algorithm between the expansion of the numerator $\mathcal{N}$ and the uncut denominators.

The inputs of the reduction algorithm implemented in NINJA are the external momenta $p_{i}$ and the masses $m_{i}$ of the loop denominators defined in eq. (2.3), and the numerator $\mathcal{N}\left(q, \mu^{2}\right)$ of the integrand cast in four different forms. 
- The first form provides a simple evaluation of the numerator as a function of $q$ and $\mu^{2}$, which is used in order to compute the coefficient $c_{0}$ of the boxes. It can also be used in order to compute the spurious coefficients of the pentagons via penta-cuts, and all the ones of the boxes when the expansion in $\mu^{2}$ is not provided.

The other three forms of the numerator yield instead the leading terms of a parametric expansion of the integrand.

- The first expansion is the one used in order to obtain the coefficient $c_{4}$ of the boxes. When the rank $r$ is equal to the number $n$ of external legs of the diagram, this method returns the coefficient of $t^{n}$ obtained by substituting in the numerator $\mathcal{N}\left(q, \mu^{2}\right)$

$$
q^{\nu} \rightarrow t v^{\nu}, \quad \mu^{2} \rightarrow t^{2} v^{2}
$$

as a function of a generic vector $v$, which is computed by NINJA and is determined by the quadruple-cut constraints.

- The second expansion is used in order to compute triangles and tadpole coefficients. In this case it returns coefficients of the terms $t^{j} \mu^{2 k}$ for $j=r, r-1, \ldots, n-3$, obtained from $\mathcal{N}\left(q, \mu^{2}\right)$ with the substitutions

$$
q^{\nu} \rightarrow v_{0}^{\nu}+t v_{3}^{\nu}+\frac{\beta+\mu^{2}}{t} v_{4}^{\nu}, \quad v_{3}^{2}=v_{4}^{2}=0
$$

as a function of the generic momenta $v_{i}^{\nu}$ and the constant $\beta_{0}$. Since in a renormalizable theory $r \leq n$, and by definition of rank we have $j+2 k \leq r$, at most 6 terms can be non-vanishing in the specified range of $j$. For effective theories with $r \leq n+1$, one can have instead up to 9 non-vanishing polynomial terms. In each call of the numerator, NinJA specifies the lowest power of $t$ which is needed in the evaluation, avoiding thus the computation of unnecessary coefficients of the expansion.

- The third and last expansion is needed for the computation of the 2-point residues and returns the coefficients of the terms $t^{j} \mu^{2 k} x^{l}$ for $j=r, r-1, \ldots, n-2$, obtained from $\mathcal{N}\left(q, \mu^{2}\right)$ with the substitutions

$$
q^{\nu} \rightarrow v_{1}^{\nu}+x v_{2}^{\nu}+t v_{3}^{\nu}+\frac{\beta_{0}+\beta_{1} x+\beta_{2} x^{2}+\mu^{2}}{t} v_{4}^{\nu}, \quad v_{2} \cdot v_{3}=v_{2} \cdot v_{4}=v_{3}^{2}=v_{4}^{2}=0
$$

as a function of the cut-dependent momenta $v_{i}^{\nu}$ and constants $\beta_{i}$. In a renormalizable theory one can have at most 7 non-vanishing terms in this range of $j$, while for $r \leq n+1$ one can have 13 non-vanishing terms. As in the previous case, in each call of the numerator, Ninja specifies the lowest power of $t$ which is needed. It is worth to notice that the expansion in eq. (2.34) can be obtained from the previous one in eq. (2.33) with the substitutions

$$
v_{0}^{\nu} \rightarrow v_{1}^{\nu}+x v_{2}^{\nu}, \quad \beta \rightarrow \beta_{0}+\beta_{1} x+\beta_{2} x^{2}, \quad v_{2} \cdot v_{3}=v_{2} \cdot v_{4}=0 .
$$


All these expansions can be easily and quickly obtained from the knowledge of the analytic dependence of the loop momentum on $q$ and $\mu^{2}$. For every phase-space point, NinJA computes the parametric solutions of all the multiple cuts, performs the Laurent expansion of the integrand via a simplified polynomial division between the expansion of the numerator and the set of the uncut denominators, and implements the subtractions at the coefficient level appearing in eqs. (2.24) and (2.31). Finally, the obtained non-spurious coefficients are multiplied by the corresponding Master Integrals in order to get the integrated result as in eq. (2.10).

The routines which compute the Master Integrals are called by NiNJA via a generic interface which allows to use any integral library implementing it, with the possibility of switching between different libraries at run-time. By default, a $\mathrm{C}++$ wrapper of the OneLoop integral library $[83,84]$ is used. This wrapper caches every computed integral allowing constant time lookups of their values from their arguments. An interface with the LoopTools $[85,86]$ library is available as well.

The NinJa library can also be used in order to compute integrals from effective and non-renomalizable theories where the rank $r$ of the numerator can exceed the number of legs by one unit. An example of this application, given in section 5, is Higgs boson production plus three jets in gluon fusion, in the effective theory defined by the infinite top-mass limit.

\section{Interfacing NiNJA with GOSAM}

The library NinJA has been interfaced with the automatic generator of one-loop amplitudes GoSAm. The latter provides NinJA with analytic expressions for the integrands of one-loop Feynman diagrams for generic processes within the Standard Model and also for Beyond Standard Model theories.

GoSAM combines automated diagram generation and algebraic manipulation [87-90] with integrand-reduction techniques $[6,63-65,73,78]$. Amplitudes are generated via Feynman diagrams, using QGRAF [87], FORM [88], Spinney [90] and HagGies [89].

After the generation of all contributing diagrams, the virtual corrections are evaluated using the $d$-dimensional integrand-level reduction method, as implemented in the library SAMURAI [62], which allows for the combined determination of both cut-constructible and rational terms at once. As an alternative, the tensorial decomposition provided by Golem95C [91-93] is also available. Such reduction, which is numerically stable but more time consuming, is employed as a rescue system. After the reduction, all relevant master integrals can be computed by means of Golem95C [93], QCDLoop [94, 95], or ONELOOP [83].

The possibility to deal with higher-rank one-loop integrals, where powers of loop momenta in the numerator exceed the number of denominators, is implemented in all three reduction programs Samurai [78, 79], Ninja and GOLEm95C [96]. Higher rank integrals can appear when computing one-loop integrals in effective-field theories, e.g. for calculations involving the effective gluon-gluon-Higgs vertex [42, 44], or when dealing with spin-2 particles [41]. 
In order to embed NINJA into the GoSAM framework, the algebraic manipulation of the integrands was adapted to generate the expansions needed by NiNJA and described in section 2.3. The numerator, in all its forms, is then optimized for fast numerical evaluation by exploiting the new features of FORM 4 [97, 98], and written in a FORTRAn90 source file which is compiled. At running time, these expressions are used as input for NinJA.

The Fortran90 module of the interface between Ninja and GoSam defines subroutines which allow to control some of the settings of NINJA directly from settings of the code that generated the virtual part of the amplitudes. Upon importing the module, the user can change the integral library used by NinJA choosing between the use of ONELoop [83] and the LoopTools $[85,86]$.

For debugging purposes, one can also ask NinJA to perform some internal test or print some information about the ongoing computation. This option allows to choose among different internal tests, namely the global $\mathcal{N}=\mathcal{N}$ test, the local $\mathcal{N}=\mathcal{N}$ tests on different cuts, or a combination of both. These tests have been described in [62]. The verbosity of the debug output can be adjusted to control the amount of details printed out in the output file, for example the final results for the finite part and the poles of the diagram, the values of the coefficients that are computed in the reduction, the values of the corresponding Master Integrals, and the results of the various internal tests.

\section{Precision tests}

Within the context of numerical and semi-numerical techniques, the problem of estimating correctly the precision of the results is of primary importance. In particular, when performing the phase space integration of the virtual contribution, it is important to assess in real time, for each phase space point, the level of precision of the corresponding one-loop matrix element.

Whenever a phase space point is found in which the quality of the result falls below a certain threshold, either the point is discarded or the evaluation of the amplitude is repeated by means of a safer, albeit less efficient procedure. Examples of such a method involve the use of higher precision routines, or in the case of GoSAM the use of traditional tensorial reconstruction of the amplitude, provided by GoLEM95C.

Various techniques for detecting points with low precision have been implemented within the different automated tools for the evaluation of one-loop virtual corrections.

A standard method which is widely employed is based on the comparison between the numerical values of the poles with their known analytic results dictated by the universal behavior of the infrared singularities. While this method is quite reliable, not all integrals which appear in the reconstruction of the amplitude give a contribution to the double and single poles. This often results in an overestimate of the precision, which might lead to keep phase space points whose finite part is less precise than what is predicted by the poles.

A different technique, which we refer to as scaling test [9], is based on the properties of scaling of scattering amplitudes when all physical scales (momenta, renormalization scale, masses) are rescaled by a common multiplicative factor $x$. As shown in [9], this method 
provides a very good correlation between the estimated precision, and the actual precision of the finite parts.

Additional methods have been proposed, within the context of integrand-reduction approaches, which target the relations between the coefficients before integration, namely the reconstructed algebraic expressions for the numerator function before integration. This method, labeled $\mathcal{N}=\mathcal{N}$ test [61, 62], can be applied to the full amplitude (global $\mathcal{N}=\mathcal{N}$ test) or individually within each residue of individual cuts (local $\mathcal{N}=\mathcal{N}$ test). The drawback of this technique comes from the fact that the test is applied at the level of individual diagrams, rather than on the final result summed over all diagrams, making the construction of a rescue system quite cumbersome.

For the precision analysis contained in this paper, we present a new simple and efficient method for the estimation of the number of digits of precision in the results, which we call rotation test. This new method exploits the invariance of the scattering amplitudes under an azimuthal rotation about the beam axis, namely the direction of the initial colliding particles.

Such a rotation, which does not affect the initial states, changes the momenta of all final particles without changing their relative position, thus reconstructing a theoretically identical process. However, the change in the values of all final state external momenta is responsible for different bases for the parametrization of the residues within the integrand reconstruction, different coefficients in front of the master integrals, as well as different numerical values when the master integrals are computed. We tested that the choice of the angle of rotation does not affect the estimate, as long as this angle is not too small.

In order to study the correlation of the error estimated by the rotation test and the exact error, we follow the strategy of ref. [9]. In particular, we generated $10^{4}$ points for the process $u \bar{d} \rightarrow W b \bar{b} g$ with massive bottom quarks, both in quadrupole and standard double precision, which we label with $A_{\text {quad }}$ and $A$ respectively, as well as the same points in double precision after performing a rotation, called $A_{\text {rot }}$.

We define the exact error $\delta_{\text {ex }}$ as

$$
\delta_{\text {ex }}=\left|\frac{A_{\text {quad }}-A}{A_{\text {quad }}}\right|,
$$

and the estimated error $\delta_{\text {rot }}$ as

$$
\delta_{\mathrm{rot}}=2\left|\frac{A_{\mathrm{rot}}-A}{A_{\mathrm{rot}}+A}\right| .
$$

In figure 1, we plot the distribution of the quantity

$$
\mathcal{C}=\frac{\log _{10}\left(\delta_{\text {rot }}\right)}{\log _{10}\left(\delta_{\text {ex }}\right)}-1
$$

evaluated for each phase space point. In the ideal case of a perfect correlation between the exact error, as estimated by the quadrupole precision result, and the error estimated by the less time-consuming rotation test, the value of $\mathcal{C}$ would be close to zero, while the spread of the distribution can provide a picture of the degree of correlation. Moreover, we observe a similar behavior for the rotation and the scaling tests. 


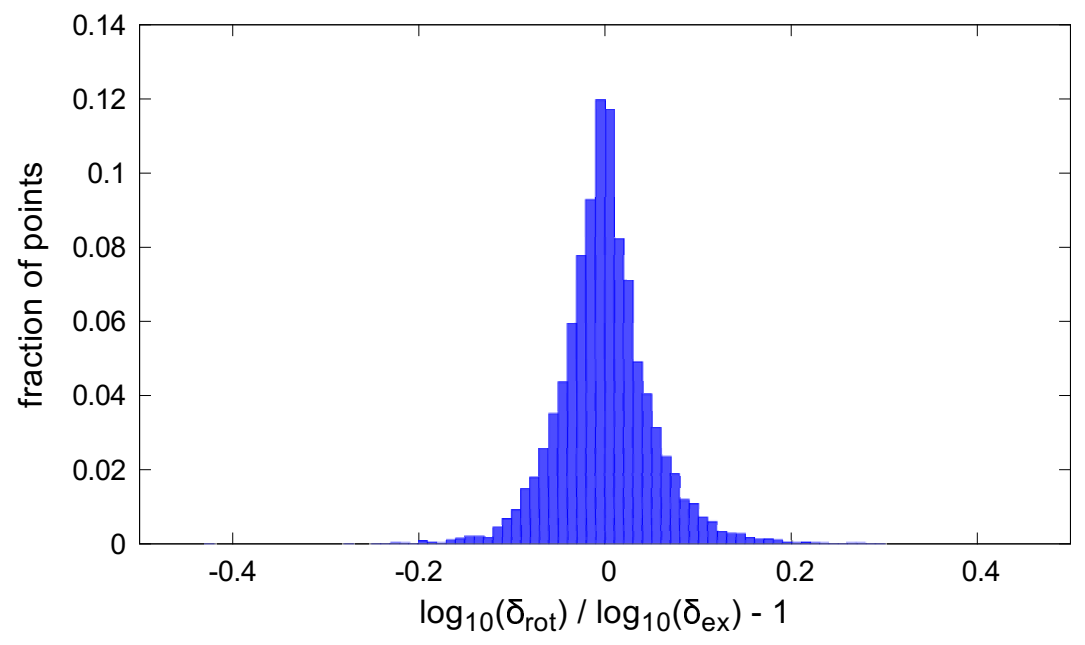

Figure 1. Correlation plot based on $10^{4}$ points for the process $u \bar{d} \rightarrow W b \bar{b} g$ with massive bottom quarks.

In the following, we will employ the rotation test as the standard method to estimate the precision of the finite part of each renormalized virtual matrix elements. If we call $\delta_{0}$ the error at any given phase space point and calculate it according to eq. (4.2), we can define the precision of the finite part as $P_{0}=\log _{10}\left(\delta_{0}\right)$. Concerning the precision of the double and single poles, $P_{-2}=\log _{10}\left(\delta_{-2}\right)$ and $P_{-1}=\log _{10}\left(\delta_{-1}\right)$, we employ the fact that the values of the pole coefficients, after renormalization, are solely due to infrared (IR) divergencies, whose expressions are well known [99]. $\delta_{-2}$ and $\delta_{-1}$ are defined using formula in eq. (4.1), in which the exact values are provided by the reconstructed IR poles, which is automatically evaluated by GoSAM.

In order to assess the level of precision of the results obtained with NiNJA within GoSAM, in figures 2 and 3, we plot the distributions of $P_{-2}$ (precision of the double pole), $P_{-1}$ (single pole) and $P_{0}$ (finite part) for two challenging virtual amplitudes with massive internal and external particles, namely $g g \rightarrow t \bar{t} H g(t \bar{t} H j)$ and $u \bar{u} \rightarrow H u \bar{u} g g(H j j j j)$ in VBF. By selecting an upper bound on the value of $P_{0}$, we can set a rejection criterium for phase space points in which the quality of the calculated scattering amplitudes falls below the requested precision. This also allows to estimate the percentage of points which would be discarded (or redirected to the rescue system). This value, as expected by analyzing the shape of the various distributions, is strongly process dependent and should be selected according to the particular phenomenological analysis at hand. As a benchmark value, in ref. [9], the threshold for rejection was set to $P_{0}=-3$. In a similar fashion, in table 1 , we provide the percentages of bad points, which are points whose precision falls below the threshold, for increasing values of the rejection threshold.

The two plots are built using a set of $5 \cdot 10^{4}$ and $1 \cdot 10^{5}$ phase space points, respectively for $g g \rightarrow t \bar{t} H g$ and $u \bar{u} \rightarrow H u \bar{u} g g(\mathrm{VBF})$. No cuts have been introduced in the selection of the points, which are randomly distributed over the whole available phase space for the outgoing particles, and are generated using RAMBO. 


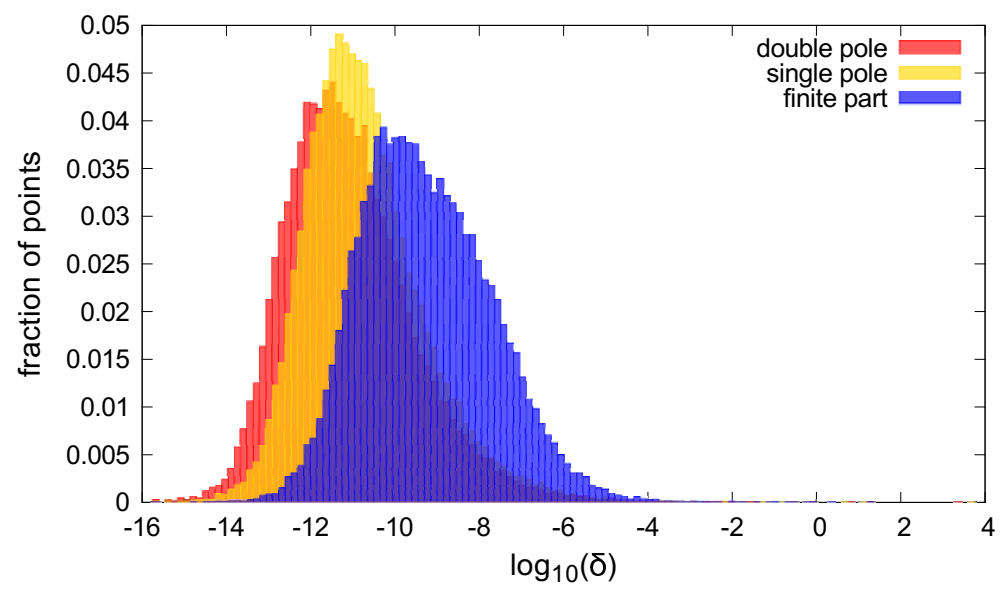

Figure 2. Precision Plot for $g g \rightarrow t \bar{t} H g$ : the distributions are obtained using $5 \cdot 10^{4}$ randomly distributed phase space points.

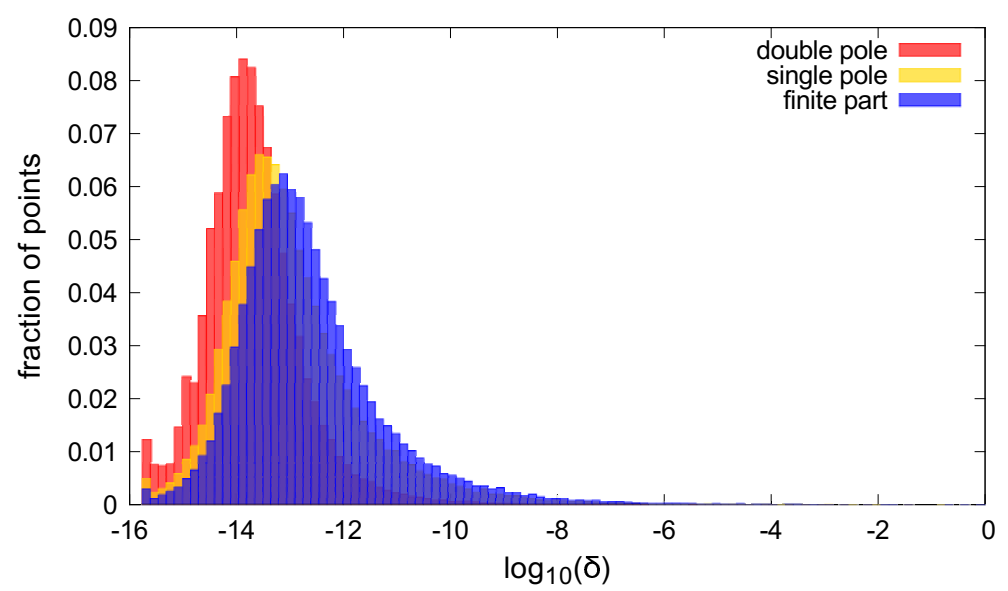

Figure 3. Precision plot for $u \bar{u} \rightarrow H u \bar{u} g g$ in VBF: the distributions are obtained using $10^{5}$ randomly distributed phase space points.

\begin{tabular}{|ccc|}
\hline$P_{0}$ & $u \bar{u} \rightarrow H u \bar{u} g g$ & $g g \rightarrow t \bar{t} H g$ \\
\hline-3 & $0.02 \%$ & $0.06 \%$ \\
-4 & $0.04 \%$ & $0.16 \%$ \\
-5 & $0.08 \%$ & $0.56 \%$ \\
\hline
\end{tabular}

Table 1. Percentage of bad points as a function of the rejection threshold $P_{0}$.

The use of the novel algorithm implemented in NiNJA yields significant improvements both in the accuracy of results and in reduction of the computational time, due to a more efficient reduction and less frequent calls to the rescue system.

These features make GoSAM+NINJA an extremely competitive framework for massive, as well as massless, calculations. The new library has been recently used in the evaluation of NLO QCD corrections to $p p \rightarrow t \bar{t} H j[45]$. 


\section{Applications to massive amplitudes}

In order to demonstrate the performances of the new reduction algorithm, we apply GoSAm+Ninja to a collection of processes involving six, seven and eight external particles. We choose processes where massive particles appear in the products of the reactions or run in the loop. We list them in table 2 , and give the details of their calculations in the following subsections: for each process we provide results for a phase space point and a detailed list of the input parameters employed. While some of the considered processes have

\begin{tabular}{|c|c|c|c|}
\hline \multicolumn{4}{|c|}{ Benchmarks: GoSAM + NinJA } \\
\hline \multicolumn{2}{|l|}{ Process } & \# NLO diagrams & ms/event \\
\hline$W+3 j$ & $d \bar{u} \rightarrow \bar{\nu}_{e} e^{-} g g g$ & 1411 & 226 \\
\hline$Z+3 j$ & $d \bar{d} \rightarrow e^{+} e^{-} g g g$ & 2928 & 1911 \\
\hline$Z Z Z+1 j$ & $u \bar{u} \rightarrow Z Z Z g$ & 915 & $* 12000$ \\
\hline$W W Z+1 j$ & $u \bar{u} \rightarrow W^{+} W^{-} Z g$ & 779 & $* 7050$ \\
\hline$W Z Z+1 j$ & $u \bar{d} \rightarrow W^{+} Z Z g$ & 756 & $* 3300$ \\
\hline$W W W+1 j$ & $u \bar{d} \rightarrow W^{+} W^{-} W^{+} g$ & 569 & $* 1800$ \\
\hline$Z Z Z Z$ & $u \bar{u} \rightarrow Z Z Z Z$ & 408 & $* 1070$ \\
\hline$W W W W$ & $u \bar{u} \rightarrow W^{+} W^{-} W^{+} W^{-}$ & 496 & *1350 \\
\hline \multirow{2}{*}{$t \bar{t} b \bar{b}\left(m_{b} \neq 0\right)$} & $d \bar{d} \rightarrow t \bar{t} b \bar{b}$ & 275 & 178 \\
\hline & $g g \rightarrow t \bar{t} b \bar{b}$ & 1530 & 5685 \\
\hline$t \bar{t}+2 j$ & $g g \rightarrow t \bar{t} g g$ & 4700 & 13827 \\
\hline$Z b \bar{b}+1 j\left(m_{b} \neq 0\right)$ & $d u g \rightarrow u e^{+} e^{-} b \bar{b}$ & 708 & $* 1070$ \\
\hline$W b \bar{b}+1 j\left(m_{b} \neq 0\right)$ & $u \bar{d} \rightarrow e^{+} \nu_{e} b \bar{b} g$ & 312 & 67 \\
\hline \multirow{3}{*}{$W b \bar{b}+2 j\left(m_{b} \neq 0\right)$} & $u \bar{d} \rightarrow e^{+} \nu_{e} b \bar{b} s \bar{s}$ & 648 & 181 \\
\hline & $u \bar{d} \rightarrow e^{+} \nu_{e} b \bar{b} d \bar{d}$ & 1220 & 895 \\
\hline & $u \bar{d} \rightarrow e^{+} \nu_{e} b \bar{b} g g$ & 3923 & 5387 \\
\hline \multirow{2}{*}{$W W b \bar{b}\left(m_{b} \neq 0\right)$} & $d \bar{d} \rightarrow \nu_{e} e^{+} \bar{\nu}_{\mu} \mu^{-} b \bar{b}$ & 292 & 115 \\
\hline & $g g \rightarrow \nu_{e} e^{+} \bar{\nu}_{\mu} \mu^{-} b \bar{b}$ & 1068 & $* 5300$ \\
\hline$W W b \bar{b}+1 j\left(m_{b}=0\right)$ & $u \bar{u} \rightarrow \nu_{e} e^{+} \bar{\nu}_{\mu} \mu^{-} b \bar{b} g$ & 3612 & $* 2000$ \\
\hline$H+3 j$ in $\mathrm{GF}$ & $g g \rightarrow H g g g$ & 9325 & 8961 \\
\hline \multirow{2}{*}{$t \bar{t} Z+1 j$} & $u \bar{u} \rightarrow t \bar{t} e^{+} e^{-} g$ & 1408 & 1220 \\
\hline & $g g \rightarrow t \bar{t} e^{+} e^{-} g$ & 4230 & 19560 \\
\hline$t \bar{t} H+1 j$ & $g g \rightarrow t \bar{t} H g$ & 1517 & 1505 \\
\hline$H+3 j$ in $\mathrm{VBF}$ & $u \bar{u} \rightarrow H g u \bar{u}$ & 432 & 101 \\
\hline$H+4 j$ in $\mathrm{VBF}$ & $u \bar{u} \rightarrow H g g u \bar{u}$ & 1176 & 669 \\
\hline$H+5 j$ in $\mathrm{VBF}$ & $u \bar{u} \rightarrow H g g g u \bar{u}$ & 15036 & 29200 \\
\hline
\end{tabular}

Table 2. A summary of results obtained with GoSAm+Ninja. Timings refer to full color- and helicity-summed amplitudes, using an Intel Core i7 CPU @ 3.40GHz, compiled with ifort. The timings indicated with an $\left(^{*}\right)$ are obtained with an $\operatorname{Intel}(\mathrm{R}) \mathrm{Xeon}(\mathrm{R}) \mathrm{CPU}$ E5-2650 0 @ 2.00GHz, compiled with gfortran. 
already been studied in the literature, the virtual NLO QCD contributions to $p p \rightarrow W b \bar{b}+n$ jets $(n=1,2), p p \rightarrow Z b \bar{b} j, p p \rightarrow Z t \bar{t} j, p p \rightarrow V V V j$ (with $V=W, Z$ ), $p p \rightarrow Z Z Z Z$, and $p p \rightarrow H+n$ jets $(n=4,5)$ in $\mathrm{VBF}$ are presented in this paper for the first time. When occurring in the final state, the bottom quark is treated as massive.

For calculation which were already performed with previous versions of the GoSAM framework, we observe that the new reduction technique yields a significant net gain both in computing time and in accuracy. A paradigmatic example is represented by $g g \rightarrow H g g g$, whose evaluation per ps-point required approximately 20 seconds, as reported in [44], while now can be computed in less than 10 seconds.

In the following, for each of the considered scattering amplitudes, we provide a benchmark phase space point for the most involved subprocesses and, when possible, a comparison with results available in the literature. The coefficients $a_{i}$ are which appear in the various tables are defined as follows:

$$
\frac{a_{-2}}{\epsilon^{2}}+\frac{a_{-1}}{\epsilon}+a_{0} \equiv \frac{2 \mathfrak{R e}\left\{\mathcal{M}^{\text {tree-level* }} \mathcal{M}^{\text {one-loop }}\right\}}{\left(\alpha_{s} / 2 \pi\right)\left|\mathcal{M}^{\text {tree-level }}\right|^{2}},
$$

where the finite part $a_{0}$ is computed in the dimensional reduction scheme if not stated otherwise. The reconstruction of the renormalized pole can be checked against the value of $a_{-1}$ and $a_{-2}$ obtained by the universal singular behavior of the dimensionally regularized one-loop amplitudes [99], while the precision of the finite parts is estimated by re-evaluating the amplitudes for a set of momenta rotated by an arbitrary angle about the axis of collision, as described in section 4. The accuracy of the results obtained with GoSAM+NinJA is indicated by the underlined digits.

\section{$5.1 \quad p p \rightarrow W+3$ jets}

Partonic process: $\boldsymbol{d} \overline{\boldsymbol{u}} \rightarrow \overline{\boldsymbol{\nu}}_{e} \boldsymbol{e}^{-} \boldsymbol{g g g}$. The finite part for this process is given in the conventional dimensional regularization (CDR) scheme and was compared to the new version of NJET [16]. We also find agreement in the phase space point given by the BLACKHAT Collaboration [22].

\begin{tabular}{|ccccc|}
\hline PARTICLE & $E$ & $p_{x}$ & $p_{y}$ & $p_{z}$ \\
\hline$p_{1}$ & 500.0000000000000000 & 0.0000000000000000 & 0.0000000000000000 & 500.0000000000000000 \\
$p_{2}$ & 500.0000000000000000 & 0.0000000000000000 & 0.0000000000000000 & -500.0000000000000000 \\
$p_{3}$ & 414.1300683745429865 & 232.1455649459389861 & 332.7544367808189918 & -82.9857518524426041 \\
$p_{4}$ & 91.8751521026383955 & -43.3570226791010995 & -24.0058236140056991 & 77.3623460793434958 \\
$p_{5}$ & 86.3540681437814044 & -15.2133893202618005 & 37.6335512949163018 & -76.2187226821854011 \\
$p_{6}$ & 280.1181818093759830 & -83.1261116505822031 & -263.2038567586509998 & 47.7490851160265990 \\
$p_{7}$ & 127.5225295696610033 & -90.4490412959934957 & -83.1783077030789002 & 34.0930433392580028 \\
\hline
\end{tabular}

\begin{tabular}{|cc|}
\hline PARAMETER & VALUE \\
\hline$m_{W}$ & $80.419 \mathrm{GeV}$ \\
$w_{W}$ & $2.0476 \mathrm{GeV}$ \\
$N_{f}$ & 5 \\
$\mu$ & $1000.0 \mathrm{GeV}$ \\
\hline
\end{tabular}

\begin{tabular}{|rrr|}
\hline & $d \bar{u} \rightarrow \bar{\nu}_{e} e^{-} g g g$ & Ref. [16] \\
\hline$a_{0}$ & $\underline{-91.1772093904611438}$ & -91.17720939055536 \\
$a_{-1}$ & $\underline{-57.6891244440692361}$ & -57.68912444409629 \\
$a_{-2}$ & $\underline{-11.66666666666668277}$ & -11.666666666666660 \\
\hline
\end{tabular}

Table 3. Benchmark point for the subprocess $d\left(p_{1}\right) \bar{u}\left(p_{2}\right) \rightarrow \bar{\nu}_{e}\left(p_{3}\right) e^{-}\left(p_{4}\right) g\left(p_{5}\right) g\left(p_{6}\right) g\left(p_{7}\right)$. 


\section{$5.2 p p \rightarrow Z+3$ jets}

Partonic process: $\boldsymbol{d} \overline{\boldsymbol{d}} \rightarrow \boldsymbol{e}^{+} \boldsymbol{e}^{-} \boldsymbol{g g g}$. The finite part for this process is given in CDR and was compared to the new version of NJET [16]. We also find agreement in the phase space point given by the BLACKHAT Collaboration [25].

\begin{tabular}{|ccccc|}
\hline PARTICLE & $E$ & $p_{x}$ & $p_{y}$ & $p_{z}$ \\
\hline$p_{1}$ & 500.0000000000000000 & 0.0000000000000000 & 0.0000000000000000 & 500.0000000000000000 \\
$p_{2}$ & 500.0000000000000000 & 0.0000000000000000 & 0.0000000000000000 & -500.0000000000000000 \\
$p_{3}$ & 414.1300683745429865 & 232.1455649459389861 & 332.7544367808189918 & -82.9857518524426041 \\
$p_{4}$ & 91.8751521026383955 & -43.3570226791010995 & -24.0058236140056991 & 77.3623460793434958 \\
$p_{5}$ & 86.3540681437814044 & -15.2133893202618005 & 37.6335512949163018 & -76.2187226821854011 \\
$p_{6}$ & 280.1181818093759830 & -83.1261116505822031 & -263.2038567586509998 & 47.7490851160265990 \\
$p_{7}$ & 127.5225295696610033 & -90.4490412959934957 & -83.1783077030789002 & 34.0930433392580028 \\
\hline
\end{tabular}

\begin{tabular}{|cc|}
\hline PARAMETER & VALUE \\
\hline$m_{W}$ & $80.419 \mathrm{GeV}$ \\
$m_{Z}$ & $91.188 \mathrm{GeV}$ \\
$w_{Z}$ & $2.4414 \mathrm{GeV}$ \\
$N_{f}$ & 5 \\
$\mu$ & $1000.0 \mathrm{GeV}$ \\
\hline
\end{tabular}

\begin{tabular}{|rrr|}
\hline & $d \bar{d} \rightarrow e^{+} e^{-} g g g$ & Ref. [16] \\
\hline$a_{0}$ & $\underline{-91.0463291277814761}$ & -91.04632919538757 \\
$a_{-1}$ & $\underline{-57.687671748094} 1892$ & -57.68767174883881 \\
$a_{-2}$ & $\underline{-11.666666666666} 9485$ & -11.66666666666667 \\
\hline
\end{tabular}

Table 4. Benchmark point for the subprocess $d\left(p_{1}\right) \bar{d}\left(p_{2}\right) \rightarrow e^{+}\left(p_{3}\right) e^{-}\left(p_{4}\right) g\left(p_{5}\right) g\left(p_{6}\right) g\left(p_{7}\right)$.

\section{$5.3 p p \rightarrow Z Z Z+1$ jet}

Partonic process: $u \bar{u} \rightarrow Z Z Z g$.

\begin{tabular}{|ccccc|}
\hline PARTICLE & $E$ & $p_{x}$ & $p_{y}$ & $p_{z}$ \\
\hline$p_{1}$ & 250.0000000000000000 & 0.0000000000000000 & 0.0000000000000000 & 250.0000000000000000 \\
$p_{2}$ & 250.0000000000000000 & 0.0000000000000000 & 0.0000000000000000 & -250.0000000000000000 \\
$p_{3}$ & 98.2984277476074197 & 25.7992145382080409 & -12.3240263933454042 & 23.0200218627010820 \\
$p_{4}$ & 178.4558180449861595 & -120.4664227955374400 & -73.7638561773535599 & -59.8166791207833768 \\
$p_{5}$ & 142.0905125919531145 & 50.7287365223860434 & 91.2424257480069656 & -31.2402050144644221 \\
$p_{6}$ & 81.1552416154533205 & 43.9384717349430645 & -5.1545431773078745 & 68.0368622725466565 \\
\hline
\end{tabular}

\begin{tabular}{|cc|}
\hline PARAMETER & VALUE \\
\hline$m_{W}$ & $80.376 \mathrm{GeV}$ \\
$m_{Z}$ & $91.1876 \mathrm{GeV}$ \\
$N_{f}$ & 5 \\
$\mu$ & $500.0 \mathrm{GeV}$ \\
\hline
\end{tabular}

\begin{tabular}{|rr|}
\hline & $u \bar{u} \rightarrow Z Z Z g$ \\
\hline$a_{0}$ & $\underline{-18.2274687669522883}$ \\
$a_{-1}$ & $\underline{-22.3832348831861125}$ \\
$a_{-2}$ & $\underline{-5.6666666666670755}$ \\
\hline
\end{tabular}

Table 5. Benchmark point for the subprocess $u\left(p_{1}\right) \bar{u}\left(p_{2}\right) \rightarrow Z\left(p_{3}\right) Z\left(p_{4}\right) Z\left(p_{5}\right) g\left(p_{6}\right)$. 
$5.4 \quad p p \rightarrow W W Z+1$ jet

Partonic process: $u \bar{u} \rightarrow W^{+} W^{-} Z g$.

\begin{tabular}{|ccccc|}
\hline PARTICLE & $E$ & $p_{x}$ & $p_{y}$ & $p_{z}$ \\
\hline$p_{1}$ & 250.0000000000000000 & 0.0000000000000000 & 0.0000000000000000 & 250.0000000000000000 \\
$p_{2}$ & 250.0000000000000000 & 0.0000000000000000 & 0.0000000000000000 & -250.0000000000000000 \\
$p_{3}$ & 89.1058761118447791 & 27.0348198946258513 & -12.9142626969235721 & 24.1225229592474193 \\
$p_{4}$ & 179.7207629659015140 & -126.2359378733789299 & -77.2966387614043384 & -62.6814876216509802 \\
$p_{5}$ & 146.1313400758695593 & 53.1582949292336409 & 95.6123118862003167 & -32.7363964816230890 \\
$p_{6}$ & 85.0420208463841476 & 46.0428230495191357 & -5.4014104278722739 & 71.2953611440265860 \\
\hline
\end{tabular}

\begin{tabular}{|cc|}
\hline PARAMETER & VALUE \\
\hline$m_{W}$ & $80.376 \mathrm{GeV}$ \\
$m_{Z}$ & $91.1876 \mathrm{GeV}$ \\
$N_{f}$ & 5 \\
$\mu$ & $500.0 \mathrm{GeV}$ \\
\hline
\end{tabular}

\begin{tabular}{|rr|}
\hline & $u \bar{u} \rightarrow W^{+} W^{-} Z g$ \\
\hline$a_{0}$ & $\underline{-18.0050305906438837}$ \\
$a_{-1}$ & $\underline{-22.1025452011781987}$ \\
$a_{-2}$ & $\underline{-5.6666666666666661}$ \\
\hline
\end{tabular}

Table 6. Benchmark point for the subprocess $u\left(p_{1}\right) \bar{u}\left(p_{2}\right) \rightarrow W^{+}\left(p_{3}\right) W^{-}\left(p_{4}\right) Z\left(p_{5}\right) g\left(p_{6}\right)$.

\section{5 $p p \rightarrow W Z Z+1$ jet}

Partonic process: $u \bar{d} \rightarrow W^{+} Z Z g$.

\begin{tabular}{|ccccc|}
\hline PARTICLE & $E$ & $p_{x}$ & $p_{y}$ & $p_{z}$ \\
\hline$p_{1}$ & 250.0000000000000000 & 0.0000000000000000 & 0.0000000000000000 & 250.0000000000000000 \\
$p_{2}$ & 250.0000000000000000 & 0.0000000000000000 & 0.0000000000000000 & -250.0000000000000000 \\
$p_{3}$ & 88.8514948513947331 & 26.6180234830689777 & -12.7151632255396141 & 23.7506254934495686 \\
$p_{4}$ & 182.6577199093957518 & -124.2897556491134168 & -76.1049547854528043 & -61.7151257515301381 \\
$p_{5}$ & 144.7598590943426586 & 52.3387523298249846 & 94.1382547757718982 & -32.2316987387113372 \\
$p_{6}$ & 83.7309261448668849 & 45.3329798362191525 & -5.3181367647793465 & 70.1961989967918498 \\
\hline
\end{tabular}

\begin{tabular}{|cc|}
\hline PARAMETER & VALUE \\
\hline$m_{W}$ & $80.376 \mathrm{GeV}$ \\
$m_{Z}$ & $91.1876 \mathrm{GeV}$ \\
$N_{f}$ & 5 \\
$\mu$ & $500.0 \mathrm{GeV}$ \\
\hline
\end{tabular}

\begin{tabular}{|rr|}
\hline$u \bar{d} \rightarrow W^{+} Z Z g$ \\
\hline$a_{0}$ & $\underline{-16.6719638614981740}$ \\
$a_{-1}$ & $\underline{-22.1957678011010735}$ \\
$a_{-2}$ & $\underline{-5.6666666666670213}$ \\
\hline
\end{tabular}

Table 7. Benchmark point for the subprocess $u\left(p_{1}\right) \bar{d}\left(p_{2}\right) \rightarrow W^{+}\left(p_{3}\right) Z\left(p_{4}\right) Z\left(p_{5}\right) g\left(p_{6}\right)$. 
5.6 $\quad p p \rightarrow W W W+1$ jet

Partonic process: $u \bar{d} \rightarrow W^{+} W^{-} W^{+} g$.

\begin{tabular}{|ccccc|}
\hline PARTICLE & $E$ & $p_{x}$ & $p_{y}$ & $p_{z}$ \\
\hline$p_{1}$ & 250.0000000000000000 & 0.0000000000000000 & 0.0000000000000000 & 250.0000000000000000 \\
$p_{2}$ & 250.0000000000000000 & 0.0000000000000000 & 0.0000000000000000 & -250.0000000000000000 \\
$p_{3}$ & 89.4258007278425993 & 27.5517039326065500 & -13.1611730250244197 & 24.5837262194154818 \\
$p_{4}$ & 182.4747913234621421 & -128.6494675939613614 & -78.7744883983191500 & -63.8799073098456347 \\
$p_{5}$ & 141.4314519821789986 & 54.1746388235997784 & 97.4403424793774207 & -33.3622900835894285 \\
$p_{6}$ & 86.6679559665162316 & 46.9231248377547274 & -5.5046810560337240 & 72.6584711740195104 \\
\hline
\end{tabular}

\begin{tabular}{|cc|}
\hline PARAMETER & VALUE \\
\hline$m_{W}$ & $80.376 \mathrm{GeV}$ \\
$m_{Z}$ & $91.1876 \mathrm{GeV}$ \\
$N_{f}$ & 5 \\
$\mu$ & $500.0 \mathrm{GeV}$ \\
\hline
\end{tabular}

\begin{tabular}{|rr|}
\hline & $u \bar{d} \rightarrow W^{+} W^{-} W^{+} g$ \\
\hline$a_{0}$ & $\underline{-15.8859769338002099}$ \\
$a_{-1}$ & $\underline{-21.9889128719035618}$ \\
$a_{-2}$ & $\underline{-5.6666666666} 864467$ \\
\hline
\end{tabular}

Table 8. Benchmark point for the subprocess $u\left(p_{1}\right) \bar{d}\left(p_{2}\right) \rightarrow W^{+}\left(p_{3}\right) W^{-}\left(p_{4}\right) W^{+}\left(p_{5}\right) g\left(p_{6}\right)$.

\section{$5.7 \quad p p \rightarrow Z Z Z Z$}

Partonic process: $u \bar{u} \rightarrow Z Z Z Z$.

\begin{tabular}{|ccccc|}
\hline PARTICLE & $E$ & $p_{x}$ & $p_{y}$ & $p_{z}$ \\
\hline$p_{1}$ & 250.0000000000000000 & 0.0000000000000000 & 0.0000000000000000 & 250.0000000000000000 \\
$p_{2}$ & 250.0000000000000000 & 0.0000000000000000 & 0.0000000000000000 & -250.0000000000000000 \\
$p_{3}$ & 96.3863867610220666 & 21.9480082963376795 & -10.4843437432888980 & 19.5836826767025762 \\
$p_{4}$ & 159.2027435644542095 & -102.4836644941604078 & -62.7526750844588079 & -50.8874782857443790 \\
$p_{5}$ & 130.0351856078737001 & 43.1561483551038094 & 77.6221118797800074 & -26.5767889104262487 \\
$p_{6}$ & 114.3756840666499812 & 37.3795078427186667 & -4.3850930520321931 & 57.8805845194680018 \\
\hline
\end{tabular}

\begin{tabular}{|cc|}
\hline PARAMETER & VALUE \\
\hline$m_{W}$ & $80.376 \mathrm{GeV}$ \\
$m_{Z}$ & $91.1876 \mathrm{GeV}$ \\
$N_{f}$ & 5 \\
$\mu$ & $500.0 \mathrm{GeV}$ \\
\hline
\end{tabular}

\begin{tabular}{|rr|}
\hline & $u \bar{u} \rightarrow Z Z Z Z$ \\
\hline$a_{0}$ & $\underline{10.0010268560339206}$ \\
$a_{-1}$ & $\underline{-3.9999999999613696}$ \\
$a_{-2}$ & $\underline{-2.6666666666665884}$ \\
\hline
\end{tabular}

Table 9. Benchmark point for the subprocess $u\left(p_{1}\right) \bar{u}\left(p_{2}\right) \rightarrow Z\left(p_{3}\right) Z\left(p_{4}\right) Z\left(p_{5}\right) Z\left(p_{6}\right)$. 


\section{$5.8 \quad p p \rightarrow W W W W$}

Partonic process: $u \bar{u} \rightarrow W^{+} W^{-} W^{+} W^{-}$.

\begin{tabular}{|ccccc|}
\hline PARTICLE & $E$ & $p_{x}$ & $p_{y}$ & $p_{z}$ \\
\hline$p_{1}$ & 250.0000000000000000 & 0.0000000000000000 & 0.0000000000000000 & 250.0000000000000000 \\
$p_{2}$ & 250.0000000000000000 & 0.0000000000000000 & 0.0000000000000000 & -250.0000000000000000 \\
$p_{3}$ & 87.7920438094441096 & 24.8207950462383629 & -11.8566452013738353 & 22.1470015595954024 \\
$p_{4}$ & 168.0494737136866092 & -115.8978071108833205 & -70.9664068759461202 & -57.5481680112689915 \\
$p_{5}$ & 132.1031656516532848 & 48.8048800986569518 & 87.7821123452778238 & -30.0554392738635485 \\
$p_{6}$ & 112.0553168252159821 & 42.2721319659877182 & -4.9590602679577351 & 65.4566057255370879 \\
\hline
\end{tabular}

\begin{tabular}{|cc|}
\hline PARAMETER & VALUE \\
\hline$m_{W}$ & $80.376 \mathrm{GeV}$ \\
$m_{Z}$ & $91.1876 \mathrm{GeV}$ \\
$N_{f}$ & 5 \\
$\mu$ & $500.0 \mathrm{GeV}$ \\
\hline
\end{tabular}

\begin{tabular}{|rr|}
\hline & $u \bar{u} \rightarrow W^{+} W^{-} W^{+} W^{-}$ \\
\hline$a_{0}$ & $\underline{7.8556327396245011}$ \\
$a_{-1}$ & $\underline{-3.99999999999} 81126$ \\
$a_{-2}$ & $\underline{-2.666666666666} 9747$ \\
\hline
\end{tabular}

Table 10. Benchmark point for the subprocess $u\left(p_{1}\right) \bar{u}\left(p_{2}\right) \rightarrow W^{+}\left(p_{3}\right) W^{-}\left(p_{4}\right) W^{+}\left(p_{5}\right) W^{-}\left(p_{6}\right)$.

\section{$5.9 p p \rightarrow t \bar{t} b \bar{b}$}

\section{Partonic process: $d \bar{d} \rightarrow t \bar{t} b \bar{b}$.}

\begin{tabular}{|ccccc|}
\hline PARTICLE & $E$ & $p_{x}$ & $p_{y}$ & $p_{z}$ \\
\hline$p_{1}$ & 250.0000000000000000 & 0.0000000000000000 & 0.0000000000000000 & 250.0000000000000000 \\
$p_{2}$ & 250.0000000000000000 & 0.0000000000000000 & 0.0000000000000000 & -250.0000000000000000 \\
$p_{3}$ & 213.1527786548405459 & -26.8458616570582116 & -117.8628562648380296 & -38.8884799556846303 \\
$p_{4}$ & 220.5509350311838546 & 61.9684848664477599 & 92.5635212096267281 & 83.2176445698946168 \\
$p_{5}$ & 42.2714703981682263 & -16.8075489037092431 & 24.9923105627744704 & -29.3620149548096769 \\
$p_{6}$ & 24.0248159158073982 & -18.3150743056803300 & 0.3070244924368429 & -14.9671496594002438 \\
\hline
\end{tabular}

\begin{tabular}{|cc|}
\hline PARAMETER & VALUE \\
\hline$m_{t}$ & $171.2 \mathrm{GeV}$ \\
$m_{b}$ & $4.2 \mathrm{GeV}$ \\
$N_{f}$ & 4 \\
$\mu$ & $500.0 \mathrm{GeV}$ \\
\hline
\end{tabular}

\begin{tabular}{|rr|}
\hline & $d \bar{d} \rightarrow t \bar{t} b \bar{b}$ \\
\hline$a_{0}$ & $\underline{154.6475673006605973}$ \\
$a_{-1}$ & $\underline{2.7409050914577211}$ \\
$a_{-2}$ & $\underline{-2.6666666666666683}$ \\
\hline
\end{tabular}

Table 11. Benchmark point for the subprocess $d\left(p_{1}\right) \bar{d}\left(p_{2}\right) \rightarrow t\left(p_{3}\right) \bar{t}\left(p_{4}\right) b\left(p_{5}\right) \bar{b}\left(p_{6}\right)$. 
Partonic process: $g g \rightarrow t \bar{t} b \bar{b}$.

\begin{tabular}{|ccccc|}
\hline PARTICLE & $E$ & $p_{x}$ & $p_{y}$ & $p_{z}$ \\
\hline$p_{1}$ & 250.0000000000000000 & 0.0000000000000000 & 0.0000000000000000 & 250.0000000000000000 \\
$p_{2}$ & 250.0000000000000000 & 0.0000000000000000 & 0.0000000000000000 & -250.0000000000000000 \\
$p_{3}$ & 194.0670578462199387 & -60.6594324624948058 & -47.3641590248774236 & -49.2915067154802884 \\
$p_{4}$ & 172.4499944124030151 & -15.6689752760792977 & -7.1446619651393677 & -11.5324581958636152 \\
$p_{5}$ & 61.9093840678718479 & 12.0715208460656545 & 23.6785835371366993 & 55.7560301833003820 \\
$p_{6}$ & 71.5735636735052054 & 64.2568868925084331 & 30.8302374528801408 & 5.0679347280435243 \\
\hline
\end{tabular}

\begin{tabular}{|cc|}
\hline PARAMETER & VALUE \\
\hline$m_{t}$ & $171.2 \mathrm{GeV}$ \\
$m_{b}$ & $4.2 \mathrm{GeV}$ \\
$N_{f}$ & 4 \\
$\mu$ & $500.0 \mathrm{GeV}$ \\
\hline
\end{tabular}

\begin{tabular}{|rr|}
\hline & $g g \rightarrow t \bar{t} b \bar{b}$ \\
\hline$a_{0}$ & $\underline{165.1250038552732349}$ \\
$a_{-1}$ & $\underline{-3.4472725930136989}$ \\
$a_{-2}$ & $\underline{-6.0000000000001563}$ \\
\hline
\end{tabular}

Table 12. Benchmark point for the subprocess $g\left(p_{1}\right) g\left(p_{2}\right) \rightarrow t\left(p_{3}\right) \bar{t}\left(p_{4}\right) b\left(p_{5}\right) \bar{b}\left(p_{6}\right)$.

\section{$\mathbf{5 . 1 0} p p \rightarrow t \bar{t}+2$ jets}

Partonic process: $\boldsymbol{g} \boldsymbol{g} \rightarrow \boldsymbol{t} \overline{\boldsymbol{t}} \boldsymbol{g} \boldsymbol{g}$. The finite part for this process is given in CDR and was compared with ref. [29].

\begin{tabular}{|ccccc|}
\hline PARTICLE & $E$ & $p_{x}$ & $p_{y}$ & $p_{z}$ \\
\hline$p_{1}$ & 2424.7465026975200999 & 0.0000000000000000 & 0.0000000000000000 & 2424.7465026975200999 \\
$p_{2}$ & 2424.7465026975200999 & 0.0000000000000000 & 0.0000000000000000 & -2424.7465026975200999 \\
$p_{3}$ & 881.9042263139403985 & -715.3340594013137661 & -475.1625187999429158 & 101.1925816377931966 \\
$p_{4}$ & 343.4841188963524132 & -24.1478321960174789 & -6.3283366075706340 & 295.5085181344487069 \\
$p_{5}$ & 1673.4634600426329598 & 21.8782679485017297 & 1000.4115637957629588 & 1341.3344089052341133 \\
$p_{6}$ & 1950.6412001421140303 & 717.6036236488295117 & -518.9207083882492952 & -1738.0355086774759457 \\
\hline
\end{tabular}

\begin{tabular}{|cc|}
\hline PARAMETER & VALUE \\
\hline$m_{t}$ & $173.3 \mathrm{GeV}$ \\
$N_{f}$ & 5 \\
$\mu$ & $173.3 \mathrm{GeV}$ \\
\hline
\end{tabular}

\begin{tabular}{|rrr|}
\hline & $g g \rightarrow t \bar{t} g g$ & Ref. [29] \\
\hline$a_{0}$ & $\underline{-93.9825428068626394}$ & -93.98254280655584 \\
$a_{-1}$ & $\underline{47.0991735298819236}$ & 47.0991735300671 \\
$a_{-2}$ & $\underline{-11.9999999999947171}$ & -11.999999999999874 \\
\hline
\end{tabular}

Table 13. Benchmark point for the subprocess $g\left(p_{1}\right) g\left(p_{2}\right) \rightarrow t\left(p_{3}\right) \bar{t}\left(p_{4}\right) g\left(p_{5}\right) g\left(p_{6}\right)$. 
$\mathbf{5 . 1 1} p p \rightarrow Z b \bar{b}+1$ jet

Partonic process: $u g \rightarrow u e^{+} e^{-} b \bar{b}$.

\begin{tabular}{|ccccc|}
\hline PARTICLE & $E$ & $p_{x}$ & $p_{y}$ & $p_{z}$ \\
\hline$p_{1}$ & 250.0000000000000000 & 0.0000000000000000 & 0.0000000000000000 & 250.0000000000000000 \\
$p_{2}$ & 250.0000000000000000 & 0.0000000000000000 & 0.0000000000000000 & -250.0000000000000000 \\
$p_{3}$ & 34.5908278264605187 & 21.2091909896352142 & -9.4555401980608202 & 25.6376353599131122 \\
$p_{4}$ & 166.7223525775519022 & -156.7623134542972991 & -48.7827423195217946 & -29.0200617028515602 \\
$p_{5}$ & 111.6942046513332798 & 30.9750523871488710 & 106.1302756897373314 & -15.8904394000814051 \\
$p_{6}$ & 84.2714416207739418 & 35.0918815486497024 & 1.4231216042880672 & 76.4890217424595988 \\
$p_{7}$ & 102.7211733238803646 & 69.4861885288632521 & -49.3151147764427051 & -57.2161559994397777 \\
\hline
\end{tabular}

\begin{tabular}{|cc|}
\hline PARAMETER & VALUE \\
\hline$m_{W}$ & $80.376 \mathrm{GeV}$ \\
$w_{W}$ & $2.124 \mathrm{GeV}$ \\
$m_{b}$ & $4.2 \mathrm{GeV}$ \\
$N_{f}$ & 4 \\
$\mu$ & $500.0 \mathrm{GeV}$ \\
\hline
\end{tabular}

\begin{tabular}{|rr|}
\hline & $u g \rightarrow u e^{+} e^{-} b \bar{b}$ \\
\hline$a_{0}$ & $\underline{145.3708954680396630}$ \\
$a_{-1}$ & $\underline{-8.3679512693708471}$ \\
$a_{-2}$ & $\underline{-5.6666666666675392}$ \\
\hline
\end{tabular}

Table 14. Benchmark point for the subprocess $u\left(p_{1}\right) g\left(p_{2}\right) \rightarrow u\left(p_{3}\right) e^{+}\left(p_{4}\right) e^{-}\left(p_{5}\right) b\left(p_{6}\right) \bar{b}\left(p_{7}\right)$.

$\mathbf{5 . 1 2} p p \rightarrow W b \bar{b}+1$ jet

Partonic process: $u \bar{d} \rightarrow e^{+} \nu_{e} b \bar{b} g$.

\begin{tabular}{|ccccc|}
\hline PARTICLE & $E$ & $p_{x}$ & $p_{y}$ & $p_{z}$ \\
\hline$p_{1}$ & 250.0000000000000000 & 0.0000000000000000 & 0.0000000000000000 & 250.0000000000000000 \\
$p_{2}$ & 250.0000000000000000 & 0.0000000000000000 & 0.0000000000000000 & -250.0000000000000000 \\
$p_{3}$ & 93.4300963683620580 & -16.6492363753179085 & -37.4579803162897420 & -83.9576413803095818 \\
$p_{4}$ & 119.9994454272237192 & 4.7053605726301706 & -100.6826015733804809 & 65.1209660949429150 \\
$p_{5}$ & 57.9859979994296282 & 9.1381348721238638 & -4.6735873988293006 & 56.9156220722767259 \\
$p_{6}$ & 104.9559149323387999 & 87.3260122226470514 & 54.3049824548373437 & -20.5728109201014071 \\
$p_{7}$ & 123.6285452726457805 & -84.5202712920831658 & 88.5091868336622554 & -17.5061358668087337 \\
\hline
\end{tabular}

\begin{tabular}{|cc|}
\hline PARAMETER & VAlue \\
\hline$m_{W}$ & $80.376 \mathrm{GeV}$ \\
$w_{W}$ & $2.124 \mathrm{GeV}$ \\
$m_{b}$ & $4.2 \mathrm{GeV}$ \\
$N_{f}$ & 4 \\
$\mu$ & $500.0 \mathrm{GeV}$ \\
\hline
\end{tabular}

\begin{tabular}{|rr|}
\hline & $u \bar{d} \rightarrow e^{+} \nu_{e} b \bar{b} g$ \\
\hline$a_{0}$ & $\underline{129.9538554864771243}$ \\
$a_{-1}$ & $\underline{-5.338568370118} 9715$ \\
$a_{-2}$ & $\underline{-5.666666666666} 8695$ \\
\hline
\end{tabular}

Table 15. Benchmark point for the subprocess $u\left(p_{1}\right) \bar{d}\left(p_{2}\right) \rightarrow e^{+}\left(p_{3}\right) \nu_{e}\left(p_{4}\right) b\left(p_{5}\right) \bar{b}\left(p_{6}\right) g\left(p_{7}\right)$. 
5.13 $p p \rightarrow W b \bar{b}+2$ jets

Partonic process: $u \bar{d} \rightarrow e^{+} \nu_{e} b \bar{b} d \bar{d}$.

\begin{tabular}{|ccccc|}
\hline PARTICLE & $E$ & $p_{x}$ & $p_{y}$ & $p_{z}$ \\
\hline$p_{1}$ & 250.0000000000000000 & 0.0000000000000000 & 0.0000000000000000 & 250.0000000000000000 \\
$p_{2}$ & 250.0000000000000000 & 0.0000000000000000 & 0.0000000000000000 & -250.0000000000000000 \\
$p_{3}$ & 125.6965187314100234 & -17.0943040170214537 & -113.3597903541534748 & 51.5456838370753374 \\
$p_{4}$ & 72.1993434263444129 & 25.0205029393394440 & 42.4573677065765622 & 52.7644913865970722 \\
$p_{5}$ & 130.5494441454287085 & -59.2470822889473183 & 116.2307891878907924 & -2.3883575291830641 \\
$p_{6}$ & 52.9433261580396106 & 45.1283306603629413 & -14.5296908876010313 & -23.1878769876905828 \\
$p_{7}$ & 99.1517346871049057 & -5.9484899951818377 & -31.1690273964595583 & -93.9370730297576273 \\
$p_{8}$ & 19.4596328516722785 & 12.1410427014482281 & 0.3703517437466808 & 15.2031323229588882 \\
\hline
\end{tabular}

\begin{tabular}{|cc|}
\hline PARAMETER & VALUE \\
\hline$m_{W}$ & $80.376 \mathrm{GeV}$ \\
$w_{W}$ & $2.124 \mathrm{GeV}$ \\
$m_{b}$ & $4.2 \mathrm{GeV}$ \\
$N_{f}$ & 4 \\
$\mu$ & $500.0 \mathrm{GeV}$ \\
\hline
\end{tabular}

\begin{tabular}{|rr|}
\hline & $u \bar{d} \rightarrow e^{+} \nu_{e} b \bar{b} d \bar{d}$ \\
\hline$a_{0}$ & $\underline{148.2499564138260837}$ \\
$a_{-1}$ & $\underline{-7.727299512216} 3879$ \\
$a_{-2}$ & $\underline{-5.3333333333331892}$ \\
\hline
\end{tabular}

Table 16. Benchmark point for the subprocess $u\left(p_{1}\right) \bar{d}\left(p_{2}\right) \rightarrow e^{+}\left(p_{3}\right) \nu_{e}\left(p_{4}\right) b\left(p_{5}\right) \bar{b}\left(p_{6}\right) d\left(p_{7}\right) \bar{d}\left(p_{8}\right)$.

Partonic process: $u \bar{d} \rightarrow e^{+} \nu_{e} b \bar{b} s \bar{s}$.

\begin{tabular}{|ccccc|}
\hline PARTICLE & $E$ & $p_{x}$ & $p_{y}$ & $p_{z}$ \\
\hline$p_{1}$ & 250.0000000000000000 & 0.0000000000000000 & 0.0000000000000000 & 250.0000000000000000 \\
$p_{2}$ & 250.0000000000000000 & 0.0000000000000000 & 0.0000000000000000 & -250.0000000000000000 \\
$p_{3}$ & 1.8312125180161738 & 1.2481975210878733 & -0.3016228634824342 & -1.3055136470806736 \\
$p_{4}$ & 132.0663362603577298 & -16.6607423420677527 & 94.8432229336175965 & -90.3808602603960196 \\
$p_{5}$ & 121.6089450080674226 & -63.6699664483805989 & -102.6429343577717077 & 13.4780898076629523 \\
$p_{6}$ & 51.8272368161295987 & -33.3143054988286877 & -36.6615737768635270 & -14.6461098360448521 \\
$p_{7}$ & 124.2305315458477253 & 116.2047315474863467 & -2.8413585495376918 & 43.8361952698163435 \\
$p_{8}$ & 68.4357378515813224 & -3.8079147792972257 & 47.6042666140377335 & 49.0181986660422169 \\
\hline
\end{tabular}

\begin{tabular}{|cc|}
\hline PARAMETER & VALUE \\
\hline$m_{W}$ & $80.376 \mathrm{GeV}$ \\
$w_{W}$ & $2.124 \mathrm{GeV}$ \\
$m_{b}$ & $4.2 \mathrm{GeV}$ \\
$N_{f}$ & 4 \\
$\mu$ & $500.0 \mathrm{GeV}$ \\
\hline
\end{tabular}

\begin{tabular}{|rr|}
\hline & $u \bar{d} \rightarrow e^{+} \nu_{e} b \bar{b} s \bar{s}$ \\
\hline$a_{0}$ & $\underline{161.1656361677729592}$ \\
$a_{-1}$ & $\underline{-1.3276262260753209}$ \\
$a_{-2}$ & $\underline{-5.3333333333332735}$ \\
\hline
\end{tabular}

Table 17. Benchmark point for the subprocess $u\left(p_{1}\right) \bar{d}\left(p_{2}\right) \rightarrow e^{+}\left(p_{3}\right) \nu_{e}\left(p_{4}\right) b\left(p_{5}\right) \bar{b}\left(p_{6}\right) s\left(p_{7}\right) \bar{s}\left(p_{8}\right)$. 
Partonic process: $u \bar{d} \rightarrow e^{+} \nu_{e} b \bar{b} g g$.

\begin{tabular}{|ccccc|}
\hline PARTICLE & $E$ & $p_{x}$ & $p_{y}$ & $p_{z}$ \\
\hline$p_{1}$ & 250.0000000000000000 & 0.0000000000000000 & 0.0000000000000000 & 250.0000000000000000 \\
$p_{2}$ & 250.0000000000000000 & 0.0000000000000000 & 0.0000000000000000 & -250.0000000000000000 \\
$p_{3}$ & 118.4116290336479551 & 49.7302976872290259 & -105.1432905838027665 & -22.2058512007951201 \\
$p_{4}$ & 9.9876328289379046 & 7.4336546931814604 & 4.5250271451401858 & 4.9007873616352553 \\
$p_{5}$ & 113.2724870353399353 & -8.8682847027615033 & 110.4231681698792471 & 23.2614225044082019 \\
$p_{6}$ & 50.3648230617062964 & -38.8899369624844553 & -31.0689344437605435 & -6.4241355543198972 \\
$p_{7}$ & 105.2876197360011901 & -28.2113391151385819 & 10.7916624062129962 & 100.8620009592996638 \\
$p_{8}$ & 102.6758083043666687 & 18.8056083999740657 & 10.4723673063308507 & -100.3942240702281055 \\
\hline
\end{tabular}

\begin{tabular}{|cc|}
\hline PARAMETER & VALUE \\
\hline$m_{W}$ & $80.376 \mathrm{GeV}$ \\
$w_{W}$ & $2.124 \mathrm{GeV}$ \\
$m_{b}$ & $4.2 \mathrm{GeV}$ \\
$N_{f}$ & 4 \\
$\mu$ & $500.0 \mathrm{GeV}$ \\
\hline
\end{tabular}

\begin{tabular}{|rr|}
\hline & $u \bar{d} \rightarrow e^{+} \nu_{e} b \bar{b} g g$ \\
\hline$a_{0}$ & $\underline{64.8935770569783301}$ \\
$a_{-1}$ & $\underline{-35.9610562256753425}$ \\
$a_{-2}$ & $\underline{-8.6666666666670285}$ \\
\hline
\end{tabular}

Table 18. Benchmark point for the subprocess $u\left(p_{1}\right) \bar{d}\left(p_{2}\right) \rightarrow e^{+}\left(p_{3}\right) \nu_{e}\left(p_{4}\right) b\left(p_{5}\right) \bar{b}\left(p_{6}\right) g\left(p_{7}\right) g\left(p_{8}\right)$.

\section{$\mathbf{5 . 1 4} p p \rightarrow W W b \bar{b}$}

Partonic process: $d \bar{d} \rightarrow \nu_{e} e^{+} \bar{\nu}_{\mu} \mu^{-} b \bar{b}$.

\begin{tabular}{|ccccc|}
\hline PARTICLE & $E$ & $p_{x}$ & $p_{y}$ & $p_{z}$ \\
\hline$p_{1}$ & 250.0000000000000000 & 0.0000000000000000 & 0.0000000000000000 & 250.0000000000000000 \\
$p_{2}$ & 250.0000000000000000 & 0.0000000000000000 & 0.0000000000000000 & -250.0000000000000000 \\
$p_{3}$ & 25.6649135887983881 & 15.7119189648809563 & -7.6256852614994211 & 18.8061776088085644 \\
$p_{4}$ & 154.5064489409292605 & -136.9768135536888565 & -46.2042967903236104 & -54.5413446032057010 \\
$p_{5}$ & 96.1603526761926730 & 22.3143342117606984 & 88.8440473781699325 & -29.2509685474019605 \\
$p_{6}$ & 61.0731578730670606 & 23.2528867537403734 & 2.3717946919774544 & 56.4234743714476039 \\
$p_{7}$ & 93.6199892430268648 & 58.9397665353324101 & -43.8226058551382351 & -57.9028973600646211 \\
$p_{8}$ & 68.9751376779857708 & 16.7579070879743455 & 6.4367458368138966 & 66.4655585304161036 \\
\hline
\end{tabular}

\begin{tabular}{|cc|}
\hline PARAMETER & VALUE \\
\hline$m_{W}$ & $80.376 \mathrm{GeV}$ \\
$m_{Z}$ & $91.1876 \mathrm{GeV}$ \\
$m_{t}$ & $171.2 \mathrm{GeV}$ \\
$m_{b}$ & $4.2 \mathrm{GeV}$ \\
$N_{f}$ & 4 \\
$\mu$ & $500.0 \mathrm{GeV}$ \\
\hline
\end{tabular}

\begin{tabular}{|rr|}
\hline & $d \bar{d} \rightarrow \nu_{e} e^{+} \bar{\nu}_{\mu} \mu^{-} b \bar{b}$ \\
\hline$a_{0}$ & $\underline{118.3990066409585751}$ \\
$a_{-1}$ & $\underline{8.5574384926230991}$ \\
$a_{-2}$ & $\underline{-2.66666666666666492}$ \\
\hline
\end{tabular}

Table 19. Benchmark point for the subprocess $d\left(p_{1}\right) \bar{d}\left(p_{2}\right) \rightarrow \nu_{e}\left(p_{3}\right) e^{+}\left(p_{4}\right) \bar{\nu}_{\mu}\left(p_{5}\right) \mu^{-}\left(p_{6}\right) b\left(p_{7}\right) \bar{b}\left(p_{8}\right)$. 
Partonic process: $g g \rightarrow \nu_{e} e^{+} \bar{\nu}_{\mu} \mu^{-} b \bar{b}$.

\begin{tabular}{|ccccc|}
\hline PARTICLE & $E$ & $p_{x}$ & $p_{y}$ & $p_{z}$ \\
\hline$p_{1}$ & 250.0000000000000000 & 0.0000000000000000 & 0.0000000000000000 & 250.0000000000000000 \\
$p_{2}$ & 250.0000000000000000 & 0.0000000000000000 & 0.0000000000000000 & -250.0000000000000000 \\
$p_{3}$ & 25.6649135887983881 & 15.7119189648809563 & -7.6256852614994211 & 18.8061776088085644 \\
$p_{4}$ & 154.5064489409292605 & -136.9768135536888565 & -46.2042967903236104 & -54.5413446032057010 \\
$p_{5}$ & 96.1603526761926730 & 22.3143342117606984 & 88.8440473781699325 & -29.2509685474019605 \\
$p_{6}$ & 61.0731578730670606 & 23.2528867537403734 & 2.3717946919774544 & 56.4234743714476039 \\
$p_{7}$ & 93.6199892430268648 & 58.9397665353324101 & -43.8226058551382351 & -57.9028973600646211 \\
$p_{8}$ & 68.9751376779857708 & 16.7579070879743455 & 6.4367458368138966 & 66.4655585304161036 \\
\hline
\end{tabular}

\begin{tabular}{|cc|}
\hline PARAMETER & VALUE \\
\hline$m_{W}$ & $80.376 \mathrm{GeV}$ \\
$m_{Z}$ & $91.1876 \mathrm{GeV}$ \\
$m_{t}$ & $171.2 \mathrm{GeV}$ \\
$m_{b}$ & $4.2 \mathrm{GeV}$ \\
$N_{f}$ & 4 \\
$\mu$ & $500.0 \mathrm{GeV}$ \\
\hline
\end{tabular}

\begin{tabular}{|rr|}
\hline & $g g \rightarrow \nu_{e} e^{+} \bar{\nu}_{\mu} \mu^{-} b \bar{b}$ \\
\hline$a_{0}$ & $\underline{27.4387494492212056}$ \\
$a_{-1}$ & $\underline{-7.9555523940773458}$ \\
$a_{-2}$ & $\underline{-5.9999999999999} 885$ \\
\hline
\end{tabular}

Table 20. Benchmark point for the subprocess $g\left(p_{1}\right) g\left(p_{2}\right) \rightarrow \nu_{e}\left(p_{3}\right) e^{+}\left(p_{4}\right) \bar{\nu}_{\mu}\left(p_{5}\right) \mu^{-}\left(p_{6}\right) b\left(p_{7}\right) \bar{b}\left(p_{8}\right)$.

\section{$\mathbf{5 . 1 5} p p \rightarrow W W b \bar{b}+1$ jet}

Partonic process: $u \bar{u} \rightarrow \nu_{e} e^{+} \bar{\nu}_{\mu} \mu^{-} b \bar{b} g$.

\begin{tabular}{|ccccc|}
\hline PARTICLE & $E$ & $p_{x}$ & $p_{y}$ & $p_{z}$ \\
\hline$p_{1}$ & 250.0000000000000000 & 0.0000000000000000 & 0.0000000000000000 & 250.0000000000000000 \\
$p_{2}$ & 250.0000000000000000 & 0.0000000000000000 & 0.0000000000000000 & -250.0000000000000000 \\
$p_{3}$ & 18.1435357791203664 & 10.6794732394995044 & -3.3652659935279523 & 14.2762644514536543 \\
$p_{4}$ & 132.6867460765342059 & -121.1435093670056773 & -5.2844663256145745 & -53.8711159925087628 \\
$p_{5}$ & 92.9820970636741606 & 17.2946496492191066 & 87.0024013115393018 & -27.8773677719967203 \\
$p_{6}$ & 46.6813566690488102 & 14.7875289839330328 & 9.6031823094958089 & 43.2233378690595345 \\
$p_{7}$ & 69.5794003816978091 & 41.1619960772432023 & -24.4397402957503331 & -50.4943772185567497 \\
$p_{8}$ & 53.6926389028252160 & 9.1521313351918430 & 14.4565277002773502 & 50.8934845655482206 \\
$p_{9}$ & 86.2342251270994211 & 28.0677300819189206 & -77.9726387064195876 & 23.8497740970007897 \\
\hline
\end{tabular}

\begin{tabular}{|cc|}
\hline PARAMETER & VALUE \\
\hline$m_{W}$ & $80.376 \mathrm{GeV}$ \\
$m_{Z}$ & $91.1876 \mathrm{GeV}$ \\
$m_{t}$ & $171.2 \mathrm{GeV}$ \\
$m_{b}$ & $0.0 \mathrm{GeV}$ \\
$N_{f}$ & 5 \\
$\mu^{2}$ & $500.0 \mathrm{GeV}$ \\
\hline
\end{tabular}

\begin{tabular}{|rr|}
\hline & $u \bar{u} \rightarrow \nu_{e} e^{+} \bar{\nu}_{\mu} \mu^{-} b \bar{b} g$ \\
\hline$a_{0}$ & $\underline{-38.5591246673060795}$ \\
$a_{-1}$ & $\underline{-32.4828496060584442}$ \\
$a_{-2}$ & $\underline{-8.3333333333334405}$ \\
\hline
\end{tabular}

Table 21. Benchmark point for the subprocess $u\left(p_{1}\right) \bar{u}\left(p_{2}\right) \rightarrow \nu_{e}\left(p_{3}\right) e^{+}\left(p_{4}\right) \bar{\nu}_{\mu}\left(p_{5}\right) \mu^{-}\left(p_{6}\right) b\left(p_{7}\right)$ $\bar{b}\left(p_{8}\right) g\left(p_{9}\right)$. 
$\mathbf{5 . 1 6} p p \rightarrow H+3$ jets

Partonic process: $g g \rightarrow H g g g$.

\begin{tabular}{|ccccc|}
\hline PARTICLE & $E$ & $p_{x}$ & $p_{y}$ & $p_{z}$ \\
\hline$p_{1}$ & 250.0000000000000000 & 0.0000000000000000 & 0.0000000000000000 & 250.0000000000000000 \\
$p_{2}$ & 250.0000000000000000 & 0.0000000000000000 & 0.0000000000000000 & -250.0000000000000000 \\
$p_{3}$ & 144.2726812297522656 & 26.1871426153409317 & -66.9976759414063139 & 3.8924965402436307 \\
$p_{4}$ & 135.5632052379070274 & 5.8338195550562180 & 26.5129120011233681 & -132.8172227574218596 \\
$p_{5}$ & 75.6651361325424006 & -19.2292152047334604 & -65.8505932559228597 & 31.9241206051040152 \\
$p_{6}$ & 144.4989773997982923 & -12.7917469656637373 & 106.3353571962057913 & 97.0006056120742102 \\
\hline
\end{tabular}

\begin{tabular}{|cc|}
\hline PARAMETER & VALUE \\
\hline$m_{H}$ & $125.0 \mathrm{GeV}$ \\
$N_{f}$ & 5 \\
$\mu$ & $500.0 \mathrm{GeV}$ \\
\hline
\end{tabular}

\begin{tabular}{|rr|}
\hline & $g g \rightarrow$ Hggg \\
\hline$a_{0}$ & $\underline{23.4307927578718953}$ \\
$a_{-1}$ & $\underline{-56.3734964424517315}$ \\
$a_{-2}$ & $\underline{-15.0000000000008757}$ \\
\hline
\end{tabular}

Table 22. Benchmark point for the subprocess $g\left(p_{1}\right) g\left(p_{2}\right) \rightarrow H\left(p_{3}\right) g\left(p_{4}\right) g\left(p_{5}\right) g\left(p_{6}\right)$.

\section{$\mathbf{5 . 1 7} p p \rightarrow Z t \bar{t}+1$ jet}

Partonic process: $u \bar{u} \rightarrow t \bar{t} e^{+} e^{-} g$.

\begin{tabular}{|ccccc|}
\hline PARTICLE & $E$ & $p_{x}$ & $p_{y}$ & $p_{z}$ \\
\hline$p_{1}$ & 250.0000000000000000 & 0.0000000000000000 & 0.0000000000000000 & 250.0000000000000000 \\
$p_{2}$ & 250.0000000000000000 & 0.0000000000000000 & 0.0000000000000000 & -250.0000000000000000 \\
$p_{3}$ & 183.2414081421947287 & -30.2337217736484156 & 32.1314578860740667 & 48.1815850690226029 \\
$p_{4}$ & 199.0327070603159996 & 74.5268539046026035 & -40.9270527537185629 & -55.4554134393922311 \\
$p_{5}$ & 70.1181125436057044 & -63.0760999348447697 & 21.5315800178266556 & 21.7794946135846281 \\
$p_{6}$ & 20.7607087314536756 & -7.2430664321972609 & -7.1983324871256098 & -18.0756472939650585 \\
$p_{7}$ & 26.8470635224299627 & 26.0260342360878454 & -5.5376526630565506 & 3.5699810507501222 \\
\hline
\end{tabular}

\begin{tabular}{|cc|}
\hline PARAMETER & VALUE \\
\hline$m_{Z}$ & $91.1876 \mathrm{GeV}$ \\
$m_{t}$ & $171.2 \mathrm{GeV}$ \\
$N_{f}$ & 5 \\
$\mu$ & $500.0 \mathrm{GeV}$ \\
\hline
\end{tabular}

\begin{tabular}{|rr|}
\hline & $u \bar{u} \rightarrow t \bar{t} e^{+} e^{-} g$ \\
\hline$a_{0}$ & $\underline{-20.4367763710913373}$ \\
$a_{-1}$ & $\underline{-25.907854281} 5554513$ \\
$a_{-2}$ & $\underline{-5.6666666665792098}$ \\
\hline
\end{tabular}

Table 23. Benchmark point for the subprocess $u\left(p_{1}\right) \bar{u}\left(p_{2}\right) \rightarrow t\left(p_{3}\right) \bar{t}\left(p_{4}\right) e^{+}\left(p_{5}\right) e^{-}\left(p_{6}\right) g\left(p_{7}\right)$. 
Partonic process: $g g \rightarrow t \bar{t} e^{+} e^{-} g$.

\begin{tabular}{|ccccc|}
\hline PARTICLE & $E$ & $p_{x}$ & $p_{y}$ & $p_{z}$ \\
\hline$p_{1}$ & 250.0000000000000000 & 0.0000000000000000 & 0.0000000000000000 & 250.0000000000000000 \\
$p_{2}$ & 250.0000000000000000 & 0.0000000000000000 & 0.0000000000000000 & -250.0000000000000000 \\
$p_{3}$ & 174.2203895522303014 & -25.0977827305029138 & -19.5610151031829993 & 5.5472629175473589 \\
$p_{4}$ & 186.7123996976260685 & -14.0800163072181022 & 56.3619207264196902 & -46.6601246640355427 \\
$p_{5}$ & 60.3016377245591073 & 38.1795332240129639 & 22.1553968884492853 & 41.0822241824339116 \\
$p_{6}$ & 18.6184873501163182 & 5.2347824612577458 & -1.6661313271933778 & -17.7895792583830961 \\
$p_{7}$ & 60.1470856754682259 & -4.2365166475497116 & -57.2901711844925998 & 17.8202168224373914 \\
\hline
\end{tabular}

\begin{tabular}{|cc|}
\hline PARAMETER & VALUE \\
\hline$m_{Z}$ & $91.1876 \mathrm{GeV}$ \\
$m_{t}$ & $171.2 \mathrm{GeV}$ \\
$N_{f}$ & 5 \\
$\mu$ & $500.0 \mathrm{GeV}$ \\
\hline
\end{tabular}

\begin{tabular}{|rr|}
\hline & $g g \rightarrow t \bar{t} e^{+} e^{-} g$ \\
\hline$a_{0}$ & $\underline{9.2826425323344441}$ \\
$a_{-1}$ & $\underline{-26.2816094048822784}$ \\
$a_{-2}$ & $\underline{-9.0000000000005702}$ \\
\hline
\end{tabular}

Table 24. Benchmark point for the subprocess $g\left(p_{1}\right) g\left(p_{2}\right) \rightarrow t\left(p_{3}\right) \bar{t}\left(p_{4}\right) e^{+}\left(p_{5}\right) e^{-}\left(p_{6}\right) g\left(p_{7}\right)$.

$\mathbf{5 . 1 8} p p \rightarrow H t \bar{t}+1$ jet

Partonic process: $g \boldsymbol{g} \rightarrow \boldsymbol{H} t \bar{t} g$.

\begin{tabular}{|ccccc|}
\hline PARTICLE & $E$ & $p_{x}$ & $p_{y}$ & $p_{z}$ \\
\hline$p_{1}$ & 250.0000000000000000 & 0.0000000000000000 & 0.0000000000000000 & 250.0000000000000000 \\
$p_{2}$ & 250.0000000000000000 & 0.0000000000000000 & 0.0000000000000000 & -250.0000000000000000 \\
$p_{3}$ & 126.3747891763443505 & 6.8355633672742222 & -3.2652801590882752 & 6.0992096455298030 \\
$p_{4}$ & 177.2234233286846745 & -31.9178657717747534 & -19.5439094615872051 & -15.8485716665707326 \\
$p_{5}$ & 174.8995128490773538 & 13.4406996200208031 & 24.1748981179500326 & -8.2771667589629576 \\
$p_{6}$ & 21.5022746458936318 & 11.6416027844796517 & -1.3657084972745175 & 18.0265287800038720 \\
\hline
\end{tabular}

\begin{tabular}{|cc|}
\hline PARAMETER & VALUE \\
\hline$m_{H}$ & $126.0 \mathrm{GeV}$ \\
$m_{t}$ & $172.5 \mathrm{GeV}$ \\
$N_{f}$ & 5 \\
$\mu$ & $500.0 \mathrm{GeV}$ \\
\hline
\end{tabular}

\begin{tabular}{|rr|}
\hline & $g g \rightarrow H t \bar{t} g$ \\
\hline$a_{0}$ & $\underline{-37.1842465451705166}$ \\
$a_{-1}$ & $\underline{-35.9217497445515619}$ \\
$a_{-2}$ & $\underline{-8.9999999999990887}$ \\
\hline
\end{tabular}

Table 25. Benchmark point for the subprocess $g\left(p_{1}\right) g\left(p_{2}\right) \rightarrow H\left(p_{3}\right) t\left(p_{4}\right) \bar{t}\left(p_{5}\right) g\left(p_{6}\right)$. 
$\mathbf{5 . 1 9} p p \rightarrow H+3$ jets (VBF)

Partonic process: $u u \rightarrow g H u u(\mathrm{VBF})$.

\begin{tabular}{|ccccc|}
\hline PARTICLE & $E$ & $p_{x}$ & $p_{y}$ & $p_{z}$ \\
\hline$p_{1}$ & 250.0000000000000000 & 0.0000000000000000 & 0.0000000000000000 & 250.0000000000000000 \\
$p_{2}$ & 250.0000000000000000 & 0.0000000000000000 & 0.0000000000000000 & -250.0000000000000000 \\
$p_{3}$ & 40.1603071333660182 & -14.0012702315405289 & 0.4395613413056457 & 37.6380324509251736 \\
$p_{4}$ & 127.7084583092647421 & -23.4171446211731009 & -10.8486559189339324 & 4.2888607196847408 \\
$p_{5}$ & 145.0573545181100599 & -109.8833468186949176 & -94.5094823127907233 & 5.9366610719649477 \\
$p_{6}$ & 187.0738800392591656 & 147.3017616714085705 & 104.9185768904190468 & -47.8635542425748710 \\
\hline
\end{tabular}

\begin{tabular}{|cc|}
\hline PARAMETER & VALUE \\
\hline$m_{H}$ & $125.0 \mathrm{GeV}$ \\
$m_{Z}$ & $91.1876 \mathrm{GeV}$ \\
$w_{Z}$ & $2.4952 \mathrm{GeV}$ \\
$N_{f}$ & 5 \\
$\mu$ & $500.0 \mathrm{GeV}$ \\
\hline
\end{tabular}

\begin{tabular}{|rr|}
\hline & $u u \rightarrow g$ Huи \\
\hline$a_{0}$ & $\underline{-94.6818287259862359}$ \\
$a_{-1}$ & $\underline{-40.89041077795} 83796$ \\
$a_{-2}$ & $\underline{-8.3333333333336821}$ \\
\hline
\end{tabular}

Table 26. Benchmark point for the subprocess $u\left(p_{1}\right) u\left(p_{2}\right) \rightarrow g\left(p_{3}\right) H\left(p_{4}\right) u\left(p_{5}\right) u\left(p_{6}\right)$.

\section{$\mathbf{5 . 2 0} p p \rightarrow H+4$ jets (VBF)}

Partonic process: $u u \rightarrow g g H u u$ (VBF).

\begin{tabular}{|c|c|c|c|c|}
\hline PARTICLE & $E$ & $p_{x}$ & $p_{y}$ & $p_{z}$ \\
\hline$p_{1}$ & 250.0000000000000000 & 00.0000000000000000 & 0.0000000000000000 & 250.0000000000000000 \\
\hline$p_{2}$ & 250.0000000000000000 & 0.0000000000000000 & 0.0000000000000000 & -250.0000000000000000 \\
\hline$p_{3}$ & 77.8540004794647871 & $1-42.9509851388533761$ & 6.0812524321357140 & -64.6488718781321126 \\
\hline$p_{4}$ & \multicolumn{2}{|c|}{$179.6868495846460405 \quad 66.2917119993839208$} & -68.8295351006103004 & 152.1685510599341171 \\
\hline$p_{5}$ & \multicolumn{2}{|c|}{$140.8511015083574875-29.7530986170501173$} & 2.6554844463192953 & -57.6344889901617350 \\
\hline$p_{6}$ & 81.8035523206006161 & $1 \quad 2.3404081739038114$ & 78.3533883805051659 & -23.3899591949725902 \\
\hline \multirow[t]{7}{*}{$p_{7}$} & 19.8044961069310155 & 4.0719635826157594 & -18.2605901583498813 & -6.4952309966676500 \\
\hline & \multicolumn{2}{|c|}{ PARAMETER VALUE } & & \\
\hline & \multirow{2}{*}{$\begin{array}{cc}m_{H} & 125.0 \mathrm{GeV} \\
m_{Z} & 91.1876 \mathrm{GeV}\end{array}$} & $125.0 \mathrm{GeV}$ & \multicolumn{2}{|c|}{$u u \rightarrow g g H u u$} \\
\hline & & $1.1876 \mathrm{GeV}$ & \multicolumn{2}{|c|}{$a_{0} \quad \underline{-85.2117220498222565}$} \\
\hline & & $2.4952 \mathrm{GeV}$ & $a_{-1} \quad \underline{-54.22632148544}$ & 450339 \\
\hline & $N_{f}$ & 5 & $\underline{-11.33333333333}$ & 333464 \\
\hline & $\mu$ & $500.0 \mathrm{GeV}$ & & \\
\hline
\end{tabular}

Table 27. Benchmark point for the subprocess $u\left(p_{1}\right) u\left(p_{2}\right) \rightarrow g\left(p_{3}\right) g\left(p_{4}\right) H\left(p_{5}\right) u\left(p_{6}\right) u\left(p_{7}\right)$. 


\section{Partonic process: $u \boldsymbol{u} \rightarrow \boldsymbol{u} \bar{u} H u u$.}

\begin{tabular}{|ccccc|}
\hline PARTICLE & $E$ & $p_{x}$ & $p_{y}$ & $p_{z}$ \\
\hline$p_{1}$ & 250.0000000000000000 & 0.0000000000000000 & 0.0000000000000000 & 250.0000000000000000 \\
$p_{2}$ & 250.0000000000000000 & 0.0000000000000000 & 0.0000000000000000 & -250.0000000000000000 \\
$p_{3}$ & 30.3977507329956786 & 18.6382269943320686 & -8.3093459176077840 & 22.5298582897146815 \\
$p_{4}$ & 146.5123801227517504 & -137.7596903034009301 & -42.8693308104768249 & -25.5022691658345515 \\
$p_{5}$ & 158.9318930425956466 & 27.2202771824319179 & 93.2652344091314376 & -13.9642109273819610 \\
$p_{6}$ & 73.9640816926485911 & 30.8380670569460271 & 1.2506117519626909 & 67.2170735086997695 \\
$p_{7}$ & 90.1938944090082799 & 61.0631190696906998 & -43.3371694330094499 & -50.2804517051979616 \\
\hline
\end{tabular}

\begin{tabular}{|cc|}
\hline PARAMETER & VALUE \\
\hline$m_{H}$ & $125.0 \mathrm{GeV}$ \\
$m_{Z}$ & $91.1876 \mathrm{GeV}$ \\
$w_{Z}$ & $2.4952 \mathrm{GeV}$ \\
$N_{f}$ & 5 \\
$\mu$ & $500.0 \mathrm{GeV}$ \\
\hline
\end{tabular}

\begin{tabular}{|rr|}
\hline & $u u \rightarrow u \bar{u} H u u$ \\
\hline$a_{0}$ & $\underline{-36.9909931379802686}$ \\
$a_{-1}$ & $\underline{-35.2029797282532968}$ \\
$a_{-2}$ & $\underline{-8.0000000000000533}$ \\
\hline
\end{tabular}

Table 28. Benchmark point for the subprocess $u\left(p_{1}\right) u\left(p_{2}\right) \rightarrow u\left(p_{3}\right) \bar{u}\left(p_{4}\right) H\left(p_{5}\right) u\left(p_{6}\right) u\left(p_{7}\right)$.

\section{$5.21 p p \rightarrow H+5$ jets (VBF)}

Partonic process: $u u \rightarrow g g g H u u$ (VBF).

\begin{tabular}{|ccccc|}
\hline PARTICLE & $E$ & $p_{x}$ & $p_{y}$ & $p_{z}$ \\
\hline$p_{1}$ & 250.0000000000000000 & 0.0000000000000000 & 0.0000000000000000 & 250.0000000000000000 \\
$p_{2}$ & 250.0000000000000000 & 0.0000000000000000 & 0.0000000000000000 & -250.0000000000000000 \\
$p_{3}$ & 24.3265597158113103 & 9.0293044031657743 & 17.9817135111430346 & -13.6715452237514459 \\
$p_{4}$ & 74.2975116145394878 & 11.5035704263332619 & 29.2267551012052458 & -67.3319009520241991 \\
$p_{5}$ & 97.6019108689663568 & -72.1835660496625877 & 4.7127971252997813 & -65.5244636825316746 \\
$p_{6}$ & 136.6365204371290929 & 23.6483158828115840 & -47.1886774719599131 & -16.0786999325225715 \\
$p_{7}$ & 120.9034677660200998 & 23.6253257672831865 & -14.6581830826285433 & 117.6632065216435592 \\
$p_{8}$ & 46.2340295975336915 & 4.3770495700687935 & 9.9255948169403965 & 44.9434032691863266 \\
\hline
\end{tabular}

\begin{tabular}{|cc|}
\hline PARAMETER & VALUE \\
\hline$m_{H}$ & $125.0 \mathrm{GeV}$ \\
$m_{Z}$ & $91.1876 \mathrm{GeV}$ \\
$w_{Z}$ & $2.4952 \mathrm{GeV}$ \\
$N_{f}$ & 5 \\
$\mu$ & $500.0 \mathrm{GeV}$ \\
\hline
\end{tabular}

\begin{tabular}{|rr|}
\hline & $u u \rightarrow$ gggHuи \\
\hline$a_{0}$ & $\underline{-164.8823178520154897}$ \\
$a_{-1}$ & $\underline{-81.4472715794169630}$ \\
$a_{-2}$ & $\underline{-14.3333333333333570}$ \\
\hline
\end{tabular}

Table 29. Benchmark point for the subprocess $u\left(p_{1}\right) u\left(p_{2}\right) \rightarrow g\left(p_{3}\right) g\left(p_{4}\right) g\left(p_{5}\right) H\left(p_{6}\right) u\left(p_{7}\right) u\left(p_{8}\right)$. 


\section{Conclusions}

The integrand reduction techniques have changed the way to perform the decomposition of scattering amplitudes in terms of independent integrals. In these approaches, the coefficients which multiply each integral can be completely determined algebraically by relying on the knowledge of the universal structure of the residues of amplitudes at each multiple cuts. The residues are irreducible polynomials in the components of the loop momenta which are not constrained by the on-shell conditions defining the cuts. The coefficients of the master integrals are a subset of the coefficients of the residues.

The generalized unitarity strategy implemented within the integrand decomposition requires to solve a triangular system, where the coefficients of the residues, hence of the master integrals, can be projected out of cuts only after removing the contributions of higher-point residues. By adding one more ingredient to this strategy, namely the Laurent series expansion of the integrand with respect to the unconstrained components of the loop momentum, we improved the system-solving strategy, that became diagonal.

We demonstrated that this novel reduction algorithm, now implemented in the computer code NinJA, and currently interfaced to the GoSAM framework, yields a very efficient and accurate evaluation of multi-particle one-loop scattering amplitudes, no matter whether massive particles go around the loop or participate to the scattering as external legs. We used GoSAM+NinJA to compute NLO corrections to a set of non-trivial processes involving up to eight particles.

The level of automation reached in less than a decade by the evaluation of scattering amplitudes at next-to-leading order has been heavily stimulated by the awareness that the methods for computing the virtual contributions were simply not sufficient, while the techniques for controlling the infrared divergencies and, finally, for performing phase-space integration were already available. Nowadays, the scenario is changed, and one-loop contributions to many multi-particle scattering reactions are waiting to be integrated. We hope that these advancements can stimulate the developments of novel methods for computing cross sections and distributions at next-to-leading-order accuracy for high-multiplicity final states.

\section{Acknowledgments}

We thank all the other members of the GoSAM project for collaboration on the common development of the code. We also would like to thank Valery Yundin for comparisons of $Z+$ 3 jets and $W+3$ jets with NJET. The work of H.v.D., G.L., P.M., and T.P. was supported by the Alexander von Humboldt Foundation, in the framework of the Sofja Kovalevskaja Award Project Advanced Mathematical Methods for Particle Physics, endowed by the German Federal Ministry of Education and Research. The work of G.O. was supported in part by the National Science Foundation under Grant PHY-1068550. G.O. also wishes to acknowledge the kind hospitality of the Max Planck Institut für Physik in Munich at several stages during the completion of this project. 
Open Access. This article is distributed under the terms of the Creative Commons Attribution License (CC-BY 4.0), which permits any use, distribution and reproduction in any medium, provided the original author(s) and source are credited.

\section{References}

[1] Z. Bern, L.J. Dixon, D.C. Dunbar and D.A. Kosower, One-loop n-point gauge theory amplitudes, unitarity and collinear limits, Nucl. Phys. B 425 (1994) 217 [hep-ph/9403226] [INSPIRE].

[2] F. Cachazo, P. Svrček and E. Witten, MHV vertices and tree amplitudes in gauge theory, JHEP 09 (2004) 006 [hep-th/0403047] [INSPIRE].

[3] R. Britto, F. Cachazo and B. Feng, New recursion relations for tree amplitudes of gluons, Nucl. Phys. B 715 (2005) 499 [hep-th/0412308] [InSPIRE].

[4] R. Britto, F. Cachazo and B. Feng, Generalized unitarity and one-loop amplitudes in $N=4$ super-Yang-Mills, Nucl. Phys. B 725 (2005) 275 [hep-th/0412103] [INSPIRE].

[5] F. del Aguila and R. Pittau, Recursive numerical calculus of one-loop tensor integrals, JHEP 07 (2004) 017 [hep-ph/0404120] [INSPIRE].

[6] G. Ossola, C.G. Papadopoulos and R. Pittau, Reducing full one-loop amplitudes to scalar integrals at the integrand level, Nucl. Phys. B 763 (2007) 147 [hep-ph/0609007] [INSPIRE].

[7] C. Berger et al., An automated implementation of on-shell methods for one-loop amplitudes, Phys. Rev. D 78 (2008) 036003 [arXiv:0803.4180] [INSPIRE].

[8] W. Giele and G. Zanderighi, On the numerical evaluation of one-loop amplitudes: the gluonic case, JHEP 06 (2008) 038 [arXiv:0805.2152] [INSPIRE].

[9] S. Badger, B. Biedermann and P. Uwer, NGluon: A Package to Calculate One-loop Multi-gluon Amplitudes, Comput. Phys. Commun. 182 (2011) 1674 [arXiv:1011.2900] [INSPIRE].

[10] G. Bevilacqua et al., HELAC-NLO, Comput. Phys. Commun. 184 (2013) 986 [arXiv: 1110.1499] [INSPIRE].

[11] V. Hirschi et al., Automation of one-loop QCD corrections, JHEP 05 (2011) 044 [arXiv: 1103.0621] [INSPIRE].

[12] G. Cullen et al., Automated One-Loop Calculations with GoSam, Eur. Phys. J. C 72 (2012) 1889 [arXiv:1111.2034] [InSPIRE].

[13] S. Agrawal, T. Hahn and E. Mirabella, FormCalc 7, J. Phys. Conf. Ser. 368 (2012) 012054 [arXiv:1112.0124] [INSPIRE].

[14] F. Cascioli, P. Maierhofer and S. Pozzorini, Scattering Amplitudes with Open Loops, Phys. Rev. Lett. 108 (2012) 111601 [arXiv:1111.5206] [INSPIRE].

[15] S. Actis, A. Denner, L. Hofer, A. Scharf and S. Uccirati, Recursive generation of one-loop amplitudes in the Standard Model, JHEP 04 (2013) 037 [arXiv:1211.6316] [INSPIRE].

[16] S. Badger, B. Biedermann, P. Uwer and V. Yundin, Numerical evaluation of virtual corrections to multi-jet production in massless QCD,

Comput. Phys. Commun. 184 (2013) 1981 [arXiv:1209.0100] [INSPIRE]. 
[17] G. Bevilacqua, M. Czakon, C. Papadopoulos, R. Pittau and M. Worek, Assault on the NLO Wishlist: $p p \rightarrow t \bar{t} b \bar{b}, J H E P 09$ (2009) 109 [arXiv:0907.4723] [INSPIRE].

[18] A. Lazopoulos, Multi-gluon one-loop amplitudes numerically, arXiv:0812.2998 [INSPIRE].

[19] W. Giele, Z. Kunszt and J. Winter, Efficient Color-Dressed Calculation of Virtual Corrections, Nucl. Phys. B 840 (2010) 214 [arXiv:0911.1962] [INSPIRE].

[20] A. van Hameren, Multi-gluon one-loop amplitudes using tensor integrals, JHEP 07 (2009) 088 [arXiv:0905.1005] [INSPIRE].

[21] C. Berger et al., Precise Predictions for $W+3$ Jet Production at Hadron Colliders, Phys. Rev. Lett. 102 (2009) 222001 [arXiv:0902.2760] [INSPIRE].

[22] C. Berger et al., Next-to-Leading Order QCD Predictions for $W+3$-Jet Distributions at Hadron Colliders, Phys. Rev. D 80 (2009) 074036 [arXiv:0907.1984] [InSPIRE].

[23] R.K. Ellis, K. Melnikov and G. Zanderighi, Generalized unitarity at work: first NLO QCD results for hadronic $W^{+} 3$ jet production, JHEP 04 (2009) 077 [arXiv:0901.4101] [INSPIRE].

[24] R.K. Ellis, K. Melnikov and G. Zanderighi, $W+3$ jet production at the Tevatron, Phys. Rev. D 80 (2009) 094002 [arXiv:0906.1445] [INSPIRE].

[25] C. Berger et al., Next-to-Leading Order QCD Predictions for $Z, \gamma^{*}+3$-Jet Distributions at the Tevatron, Phys. Rev. D 82 (2010) 074002 [arXiv: 1004.1659] [INSPIRE].

[26] G. Bevilacqua, M. Czakon, A. van Hameren, C.G. Papadopoulos and M. Worek, Complete off-shell effects in top quark pair hadroproduction with leptonic decay at next-to-leading order, JHEP 02 (2011) 083 [arXiv: 1012.4230] [INSPIRE].

[27] A. Denner, S. Dittmaier, S. Kallweit and S. Pozzorini, NLO QCD corrections to WWbb production at hadron colliders, Phys. Rev. Lett. 106 (2011) 052001 [arXiv:1012.3975] [INSPIRE].

[28] T. Melia, K. Melnikov, R. Rontsch and G. Zanderighi, $N L O$ QCD corrections for $W^{+} W^{-}$ pair production in association with two jets at hadron colliders, Phys. Rev. D 83 (2011) 114043 [arXiv: 1104.2327] [INSPIRE].

[29] G. Bevilacqua, M. Czakon, C. Papadopoulos and M. Worek, Hadronic top-quark pair production in association with two jets at Next-to-Leading Order QCD,

Phys. Rev. D 84 (2011) 114017 [arXiv:1108.2851] [inSPIRE].

[30] N. Greiner, A. Guffanti, T. Reiter and J. Reuter, NLO QCD corrections to the production of two bottom-antibottom pairs at the LHC, Phys. Rev. Lett. 107 (2011) 102002 [arXiv: 1105.3624] [INSPIRE].

[31] F. Campanario, C. Englert, M. Rauch and D. Zeppenfeld, Precise predictions for $W \gamma \gamma+$ jet production at hadron colliders, Phys. Lett. B 704 (2011) 515 [arXiv:1106.4009] [INSPIRE].

[32] A. Denner, S. Dittmaier, S. Kallweit and S. Pozzorini, NLO QCD corrections to off-shell top-antitop production with leptonic decays at hadron colliders, JHEP 10 (2012) 110 [arXiv: 1207.5018] [INSPIRE].

[33] N. Greiner, G. Heinrich, P. Mastrolia, G. Ossola, T. Reiter et al., NLO QCD corrections to the production of $W^{+} W^{-}$plus two jets at the LHC, Phys. Lett. B 713 (2012) 277 [arXiv: 1202.6004] [INSPIRE].

[34] G. Cullen, N. Greiner and G. Heinrich, Susy-QCD corrections to neutralino pair production in association with a jet, Eur. Phys. J. C 73 (2013) 2388 [arXiv:1212.5154] [INSPIRE]. 
[35] F. Campanario, Q. Li, M. Rauch and M. Spira, $Z Z+$ jet production via gluon fusion at the LHC, JHEP 06 (2013) 069 [arXiv:1211.5429] [INSPIRE].

[36] G. Bevilacqua and M. Worek, Constraining BSM Physics at the LHC: Four top final states with NLO accuracy in perturbative QCD, JHEP 07 (2012) 111 [arXiv:1206.3064] [INSPIRE].

[37] T. Gehrmann, N. Greiner and G. Heinrich, Photon isolation effects at $N L O$ in $\gamma \gamma+$ jet final states in hadronic collisions, JHEP 06 (2013) 058 [arXiv: 1303.0824] [INSPIRE].

[38] F. Campanario, M. Kerner, L.D. Ninh and D. Zeppenfeld, WZ production in association with two jets at NLO in QCD, Phys. Rev. Lett. 111 (2013) 052003 [arXiv:1305.1623] [INSPIRE].

[39] F. Campanario and M. Kubocz, Higgs boson production in association with three jets via gluon fusion at the LHC: Gluonic contributions, Phys. Rev. D 88 (2013) 054021 [arXiv:1306.1830] [INSPIRE].

[40] G. Bevilacqua, M. Czakon, M. Krmer, M. Kubocz and M. Worek, Quantifying quark mass effects at the LHC: A study of $p p \rightarrow b \bar{b} b \bar{b}+X$ at next-to-leading order, JHEP 07 (2013) 095 [arXiv:1304.6860] [INSPIRE].

[41] N. Greiner, G. Heinrich, J. Reichel and J.F. von Soden-Fraunhofen, NLO QCD Corrections to Diphoton Plus Jet Production through Graviton Exchange, JHEP 11 (2013) 028 [arXiv:1308.2194] [INSPIRE].

[42] H. van Deurzen et al., NLO QCD corrections to the production of Higgs plus two jets at the LHC, Phys. Lett. B 721 (2013) 74 [arXiv:1301.0493] [INSPIRE].

[43] T. Gehrmann, N. Greiner and G. Heinrich, Precise QCD predictions for the production of a photon pair in association with two jets, Phys. Rev. Lett. 111 (2013) 222002 [arXiv: 1308.3660] [INSPIRE].

[44] G. Cullen et al., NLO QCD corrections to Higgs boson production plus three jets in gluon fusion, Phys. Rev. Lett. 111 (2013) 131801 [arXiv:1307.4737] [INSPIRE].

[45] H. van Deurzen et al., NLO QCD corrections to Higgs boson production in association with a top quark pair and a jet, Phys. Rev. Lett. 111 (2013) 171801 [arXiv:1307.8437] [INSPIRE].

[46] F. Campanario et al., Electroweak Higgs plus Three Jet Production at NLO QCD, Phys. Rev. Lett. 111 (2013) 211802 [arXiv:1308.2932] [INSPIRE].

[47] F. Campanario, M. Kerner, L.D. Ninh and D. Zeppenfeld, Next-to-leading order QCD corrections to $W^{+} W^{+}$and $W^{-} W^{-}$production in association with two jets, arXiv:1311.6738 [INSPIRE].

[48] M.J. Dolan, C. Englert, N. Greiner and M. Spannowsky, Further on up the road: hhjj production at the LHC, Phys. Rev. Lett. 112 (2014) 101802 [arXiv:1310.1084] [INSPIRE].

[49] S. Badger, A. Guffanti and V. Yundin, Next-to-leading order QCD corrections to di-photon production in association with up to three jets at the Large Hadron Collider, arXiv: 1312.5927 [INSPIRE].

[50] G. Heinrich, A. Maier, R. Nisius, J. Schlenk and J. Winter, NLO QCD corrections to WWbb production with leptonic decays in the light of top quark mass and asymmetry measurements,

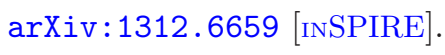

[51] P. Mastrolia, E. Mirabella, G. Ossola and T. Peraro, Scattering Amplitudes from Multivariate Polynomial Division, Phys. Lett. B 718 (2012) 173 [arXiv:1205.7087] [INSPIRE]. 
[52] Y. Zhang, Integrand-Level Reduction of Loop Amplitudes by Computational Algebraic Geometry Methods, JHEP 09 (2012) 042 [arXiv: 1205.5707] [INSPIRE].

[53] P. Mastrolia and G. Ossola, On the Integrand-Reduction Method for Two-Loop Scattering Amplitudes, JHEP 11 (2011) 014 [arXiv:1107.6041] [INSPIRE].

[54] S. Badger, H. Frellesvig and Y. Zhang, Hepta-cuts of two-loop scattering amplitudes, JHEP 04 (2012) 055 [arXiv: 1202.2019] [INSPIRE].

[55] R.H. Kleiss, I. Malamos, C.G. Papadopoulos and R. Verheyen, Counting to one: reducibility of one- and two-loop amplitudes at the integrand level, JHEP 12 (2012) 038 [arXiv: 1206.4180] [INSPIRE].

[56] S. Badger, H. Frellesvig and Y. Zhang, An integrand reconstruction method for three-loop amplitudes, JHEP 08 (2012) 065 [arXiv:1207.2976] [INSPIRE].

[57] B. Feng and R. Huang, The classification of two-loop integrand basis in pure four-dimension, JHEP 02 (2013) 117 [arXiv: 1209.3747] [INSPIRE].

[58] P. Mastrolia, E. Mirabella, G. Ossola and T. Peraro, Integrand-Reduction for Two-Loop Scattering Amplitudes through Multivariate Polynomial Division, Phys. Rev. D 87 (2013) 085026 [arXiv: 1209.4319] [INSPIRE].

[59] R. Huang and Y. Zhang, On genera of curves from high-loop generalized unitarity cuts, JHEP 04 (2013) 080 [arXiv: 1302.1023] [INSPIRE].

[60] P. Mastrolia, E. Mirabella, G. Ossola and T. Peraro, Multiloop Integrand Reduction for Dimensionally Regulated Amplitudes, Phys. Lett. B 727 (2013) 532 [arXiv:1307.5832] [INSPIRE].

[61] G. Ossola, C.G. Papadopoulos and R. Pittau, CutTools: a program implementing the OPP reduction method to compute one-loop amplitudes, JHEP 03 (2008) 042 [arXiv:0711.3596] [INSPIRE].

[62] P. Mastrolia, G. Ossola, T. Reiter and F. Tramontano, Scattering AMplitudes from Unitarity-based Reduction Algorithm at the Integrand-level, JHEP 08 (2010) 080 [arXiv: 1006.0710] [INSPIRE].

[63] G. Ossola, C.G. Papadopoulos and R. Pittau, Numerical evaluation of six-photon amplitudes, JHEP 07 (2007) 085 [arXiv:0704.1271] [INSPIRE].

[64] P. Mastrolia, G. Ossola, C. Papadopoulos and R. Pittau, Optimizing the reduction of one-loop amplitudes, JHEP 06 (2008) 030 [arXiv:0803.3964] [INSPIRE].

[65] G. Ossola, C.G. Papadopoulos and R. Pittau, On the Rational Terms of the one-loop amplitudes, JHEP 05 (2008) 004 [arXiv:0802.1876] [INSPIRE].

[66] P. Draggiotis, M. Garzelli, C. Papadopoulos and R. Pittau, Feynman Rules for the Rational Part of the QCD 1-loop amplitudes, JHEP 04 (2009) 072 [arXiv: 0903.0356] [INSPIRE].

[67] M. Garzelli, I. Malamos and R. Pittau, Feynman rules for the rational part of the Electroweak 1-loop amplitudes, JHEP 01 (2010) 040 [Erratum ibid. 1010 (2010) 097] [arXiv:0910.3130] [INSPIRE].

[68] M. Garzelli, I. Malamos and R. Pittau, Feynman rules for the rational part of the Electroweak 1-loop amplitudes in the $R_{\xi}$ gauge and in the Unitary gauge, JHEP 01 (2011) 029 [arXiv: 1009.4302] [INSPIRE]. 
[69] M. Garzelli and I. Malamos, R2SM: A Package for the analytic computation of the $R_{2}$ Rational terms in the Standard Model of the Electroweak interactions, Eur. Phys. J. C 71 (2011) 1605 [arXiv: 1010.1248] [INSPIRE].

[70] H.-S. Shao, Y.-J. Zhang and K.-T. Chao, Feynman Rules for the Rational Part of the Standard Model One-loop Amplitudes in the 't Hooft-Veltman $\gamma_{5}$ Scheme, JHEP 09 (2011) 048 [arXiv: 1106.5030] [INSPIRE].

[71] H.-S. Shao and Y.-J. Zhang, Feynman Rules for the Rational Part of One-loop QCD Corrections in the MSSM, JHEP 06 (2012) 112 [arXiv:1205.1273] [INSPIRE].

[72] B. Page and R. Pittau, $R_{2}$ vertices for the effective ggH theory, JHEP 09 (2013) 078 [arXiv: 1307.6142] [INSPIRE].

[73] R.K. Ellis, W. Giele and Z. Kunszt, A numerical unitarity formalism for evaluating one-loop amplitudes, JHEP 03 (2008) 003 [arXiv:0708.2398] [INSPIRE].

[74] W.T. Giele, Z. Kunszt and K. Melnikov, Full one-loop amplitudes from tree amplitudes, JHEP 04 (2008) 049 [arXiv:0801.2237] [INSPIRE].

[75] R.K. Ellis, W.T. Giele, Z. Kunszt and K. Melnikov, Masses, fermions and generalized D-dimensional unitarity, Nucl. Phys. B 822 (2009) 270 [arXiv:0806.3467] [INSPIRE].

[76] K. Melnikov and M. Schulze, NLO QCD corrections to top quark pair production in association with one hard jet at hadron colliders, Nucl. Phys. B 840 (2010) 129 [arXiv: 1004.3284] [INSPIRE].

[77] P. Mastrolia, E. Mirabella, G. Ossola, T. Peraro and H. van Deurzen, The Integrand Reduction of One- and Two-Loop Scattering Amplitudes, PoS(LL2012) 028 [arXiv: 1209.5678] [INSPIRE].

[78] P. Mastrolia, E. Mirabella and T. Peraro, Integrand reduction of one-loop scattering amplitudes through Laurent series expansion, JHEP 06 (2012) 095 [Erratum ibid. 1211 (2012) 128] [arXiv: 1203.0291] [INSPIRE].

[79] H. van Deurzen, Associated Higgs Production at NLO with GoSam, Acta Phys. Polon. B 44 (2013) 2223 [inSPIRE].

[80] D. Forde, Direct extraction of one-loop integral coefficients, Phys. Rev. D 75 (2007) 125019 [arXiv: 0704.1835] [INSPIRE].

[81] S. Badger, Direct extraction of one loop rational terms, JHEP 01 (2009) 049 [arXiv:0806.4600] [INSPIRE].

[82] T. Peraro, Integrand-level Reduction at One and Higher Loops, Acta Phys. Polon. B 44 (2013) 2215.

[83] A. van Hameren, OneLOop: For the evaluation of one-loop scalar functions, Comput. Phys. Commun. 182 (2011) 2427 [arXiv:1007.4716] [InSPIRE].

[84] A. van Hameren, C. Papadopoulos and R. Pittau, Automated one-loop calculations: A Proof of concept, JHEP 09 (2009) 106 [arXiv:0903.4665] [INSPIRE].

[85] T. Hahn and M. Pérez-Victoria, Automatized one loop calculations in four-dimensions and D-dimensions, Comput. Phys. Commun. 118 (1999) 153 [hep-ph/9807565] [INSPIRE].

[86] T. Hahn, Feynman Diagram Calculations with FeynArts, FormCalc and LoopTools, PoS (ACAT2010) 078 [arXiv: 1006.2231] [INSPIRE]. 
[87] P. Nogueira, Automatic Feynman graph generation, J. Comput. Phys. 105 (1993) 279 [INSPIRE].

[88] J. Vermaseren, New features of FORM, math-ph/0010025 [INSPIRE].

[89] T. Reiter, Optimising Code Generation with haggies, Comput. Phys. Commun. 181 (2010) 1301 [arXiv:0907.3714] [inSPIRE].

[90] G. Cullen, M. Koch-Janusz and T. Reiter, Spinney: A Form Library for Helicity Spinors, Comput. Phys. Commun. 182 (2011) 2368 [arXiv:1008.0803] [InSPIRE].

[91] T. Binoth, J.-P. Guillet, G. Heinrich, E. Pilon and T. Reiter, Golem95: a numerical program to calculate one-loop tensor integrals with up to six external legs,

Comput. Phys. Commun. 180 (2009) 2317 [arXiv:0810.0992] [INSPIRE].

[92] G. Heinrich, G. Ossola, T. Reiter and F. Tramontano, Tensorial reconstruction at the integrand level, JHEP 10 (2010) 105 [arXiv: 1008.2441] [INSPIRE].

[93] G. Cullen et al., Golem95C: A library for one-loop integrals with complex masses, Comput. Phys. Commun. 182 (2011) 2276 [arXiv:1101.5595] [INSPIRE].

[94] G. van Oldenborgh, FF: a package to evaluate one loop Feynman diagrams, Comput. Phys. Commun. 66 (1991) 1 [INSPIRE].

[95] R.K. Ellis and G. Zanderighi, Scalar one-loop integrals for QCD, JHEP 02 (2008) 002 [arXiv:0712.1851] [INSPIRE].

[96] J.P. Guillet, G. Heinrich and J. von Soden-Fraunhofen, Tools for NLO automation: extension of the golem95C integral library, arXiv:1312.3887 [INSPIRE].

[97] J. Kuipers, T. Ueda, J. Vermaseren and J. Vollinga, FORM version 4.0, Comput. Phys. Commun. 184 (2013) 1453 [arXiv:1203.6543] [INSPIRE].

[98] J. Kuipers, T. Ueda and J. Vermaseren, Code optimization in FORM, arXiv:1310.7007 [INSPIRE].

[99] S. Catani, S. Dittmaier and Z. Trócsányi, One loop singular behavior of QCD and SUSY QCD amplitudes with massive partons, Phys. Lett. B 500 (2001) 149 [hep-ph/0011222] [INSPIRE]. 